Rodrigo Kenji Nakagawa

\title{
O gênero Triportheus Cope 1872 (Teleostei; Characiformes; Characidae): aspectos taxonômicos, distribuição geográfica e estudo da musculatura cefálica
}


Rodrigo Kenji Nakagawa

\section{O gênero Triportheus Cope 1872 (Teleostei; Characiformes; Characidae): aspectos taxonômicos, distribuição geográfica e estudo da musculatura cefálica}

Dissertação apresentada ao Instituto de Biociências da Universidade de São Paulo, para a obtenção de Título de Mestre em Ciências Biológicas, na Área de Zoologia.

Orientador: Prof. Dra. Mônica de Toledo-Piza Ragazzo

São Paulo

2011 
Nakagawa, Rodrigo Kenji

O gênero Triportheus Cope 1872 (Teleostei; Characiformes; Characidae): aspectos taxonômicos, distribuição geográfica e estudo da musculatura cefálica

\section{$133 \mathrm{pp}$.}

Dissertação (Mestrado) - Instituto de Biociências da Universidade de São Paulo. Departamento de Zoologia.

1. Triportheus 2. Distribuição geográfica 3. Miologia

I. Universidade de São Paulo, Instituto de Biociências. Departamento de Zoologia

Comissão Julgadora: 


\section{Advertência}

Esta dissertação de mestrado não se constitui em uma publicação científica. Consequentemente, os novos dados, opiniões e hipóteses aqui apresentados não estão disponíveis na literatura zoológica. Pede-se que qualquer referência ao conteúdo desta dissertação seja feita mediante consulta prévia ao autor.

\section{Notice}

This dissertation does not constitute a scientific publication. Therefore, new data, opinions and hypothesis herein presented are not avaiable in the scientific literature. It is urged that any public reference to the contents of the present dissertation be made only after correspondence with the author. 
"Things are not always what they seem. The first appearance deceives many. The intelligence of a few perceives what has been carefully hidden."

Phaedrus 


\section{AGRADECIMENTOS}

Em primeiro lugar, gostaria de agradecer a Mônica de Toledo-Piza Ragazzo, não só pela orientação, mas pela amizade, paciência e oportunidades dadas a mim desde minha iniciação científica, anos atrás, quando eu era apenas um aluno tentando se formar. Levarei para minha vida muito mais do que conhecimentos ictiológicos de nossa convivência.

Agradeço ao Antônio Carlos Marques e ao Marcelo Rodrigues de Carvalho por cuidarem da enfadonha parte burocrática da pós-graduação e pela verba disponibilizada para compras e viagens necessárias para realização desse trabalho. Agradeço ao Conselho Nacional de Desenvolvimento Científico e Tecnológico (CNPq) pela bolsa concedida.

Agradeço ao Osvaldo T. Oyakawa por me receber no Museu de Zoologia da Universidade de São Paulo e por me dar grande liberdade de usar as instalações do museu sempre que necessário. Um obrigado para José Lima de Figueiredo, Naércio A. Menezes, Heraldo A. Britski, Mário C. C. de Pinna e a todos os alunos da ictiologia do MZUSP por sempre me acolherem em minhas visitas, e um obrigado especial para Rodrigo Caires pelas ajudas, conversas e divertidíssimas histórias.

Agradeço ao Marcelo R. de Britto (MNRJ) e ao Wolmar B. Wosiacki (MPEG) pelo envio de material importantíssimo para esse trabalho. Agradeço ao Marcelo S. Rocha (INPA) por permitir visitar a coleção mesmo com a parte de Characiformes sendo reformada. Agradeço também a Maria Cláudia Malabarba pela conversa e pelas dicas durante o EBI de Manaus.

Agradeço ao Ralf Britz pelo maravilhoso curso no qual não aprendi somente sobre anatomia de peixes, mas também como ser uma pessoa melhor. Agradeço também a todos que conheci nesse já lendário curso pela convivência e amizade. Agradeço também ao Marcelo Rodrigues de Carvalho pelo curso de biologia comparada, o qual mudou meu conceito sobre diversos tópicos acerca da sistemática e evolução.

Agradeço a todo o pessoal do laboratório de ictiologia do IBUSP pela amizade, conversas, ajudas e por tornar sempre agradável nosso ambiente de trabalho. Um obrigado muito especial para André Casas, que além de grande amigo, me ensinou o "beabá" da dissecção anatômica e me ajudou muito com as fotografias. Sem você, esse trabalho não iria existir. 
Agradeço a minha família por toda minha formação intelectual e pelo apoio que me deram nessa carreira tão difícil e subvalorizada nesse país.

Agradeço aos meus amigos Bárbara Tomotani, Carla Verna, João Tomotani e Rodrigo Salvador por me ajudarem a manter meu nível basal de (in)sanidade mental durante esses longos dois anos através de nossas atividades lúdicas de sábado à tarde.

E por fim, um obrigado muito especial para Karen Lúcia Mayumi Sasaki. Na verdade não existe agradecimento suficiente para retribuir tudo que você fez. Sua ajuda não se restringe apenas à confecção das ilustrações desse trabalho, ou à edição das imagens. Sua amizade, paciência e companheirismo me trouxeram até aqui. É por você que eu tento sempre ser uma pessoa melhor. 
O presente estudo é dividido em duas partes. Na primeira foram abordados aspectos taxonômicos das espécies do gênero Triportheus. Na revisão taxonômica mais recente do gênero, Malabarba (2004) redescreveu 13 espécies já conhecidas e descreveu 3 novas. Segundo a autora, Triportheus. angulatus e T. rotundatus ocorrem em simpatria e apresentam uma sobreposição nas características usadas como diagnósticas para cada uma delas. $\mathrm{O}$ exame de material adicional, não incluído no trabalho de Malabarba (2004), mostrou que essas características diagnósticas são consistentes para o reconhecimento de duas espécies distintas. Os sintipos de Triportheus curtus é proveniente da Ilha de Marajó e, de acordo com Malabarba (2004) todo o material não tipo é proveniente do Alto Amazonas. O presente estudo examinou 32 exemplares provenientes da Ilha de Marajó e todos eles foram identificados como $T$. rotundatus, indicando um possível problema taxonômico envolvendo essa espécie. O exame de material proveniente principalmente dos rios da Amazônia brasileira possibilitou preencher grande parte dos hiatos existentes na informação sobre as distribuições geográficas das espécies de Triportheus. Uma espécie nova é aqui descrita ocorrendo no rio Xingu. Uma chave de identificação para as espécies de Triportheus é fornecida. A segunda parte do trabalho inclui uma descrição da musculatura cefálica das espécies de Triportheus. A grande parte dos músculos se mostrou conservada para todas as espécies examinadas. O levator arcus palatini da maioria das espécies se insere apenas no hiomandibular, mas em T. albus, T. auritus e T. guentheri, o músculo também se insere no metapterigoide. O levator operculi da maioria das espécies se origina apenas no pterótico, mas em T. angulatus, T. pantanensis, T. rotundatus, T. signatus e T. trifurcatus, algumas fibras se originam no extraescapular. 


\begin{abstract}
The present work is divided in two parts. In the first, a study about some taxonomic aspects of the species of the genus Triportheus was conducted. In the most recent taxonomic revision of the genus, Malabarba (2004) redescribed 13 already known species and described 3 new ones. According to that autor, Triportheus angulatus and $T$. rotundatus occur in sympatry and there is overlap in the characteristics used as diagnostic for each species. Examination of additional material, not included in the study of Malabarba (2004), showed that those diagnostic features are consistent with the recognition of two distinct species. The syntypes of Triportheus curtus are from Ilha de Marajó, in the state of Pará, and according to Malabarba (2004) all the non-type material is from the upper Amazon. In the present study, 32 specimens of Triportheus were examined from Ilha de Marajó, and all of them were identified as $T$. rotundatus indicating a possible taxonomic problem involving this species. With examination of material mainly from rivers of the Brazilian Amazon most gaps previously present in the information of the geographic distribution of the species of Triportheus were filled. A new species is herein described from the Rio Xingu. A key to the identification of Triporhteus species is presented. In the second part of this study a description of the cephalic musculature of the species of Triportheus was conducted. Most of the muscles showed little or no variation in all species examined. The levator arcus palatini in most species inserts only in the hyomandibular, but in T. albus, T. auritus and T. guentheri, this muscle also inserts in the metapterygoid. The levator operculi of most species originates only in the pterotic, but in T. angulatus, T. pantanensis, T. rotundatus, T. signatus and $T$. trifurcatus, some fibers originate in the extrascapular.
\end{abstract}




\section{ÍNDICE}

Resumo viii

Abstract ix

Parte 1 - Aspectos taxonômicos e distribuição geográfica das espécies de Triportheus







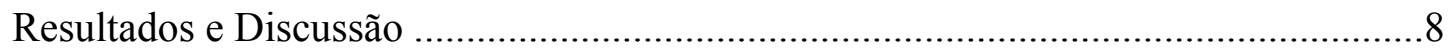

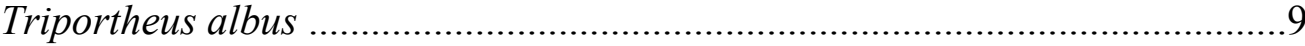



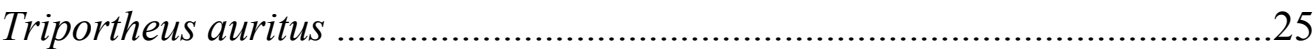

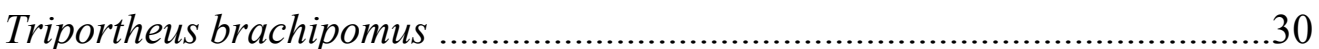



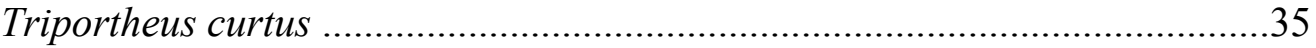

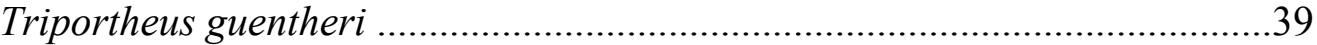

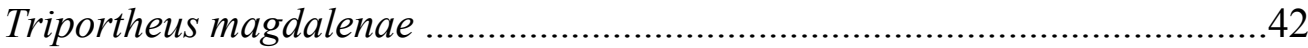

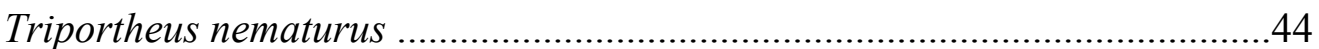

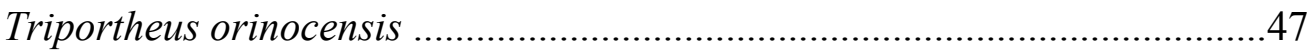



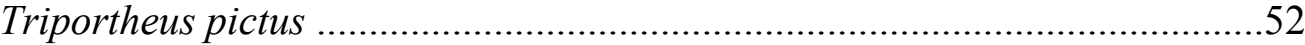

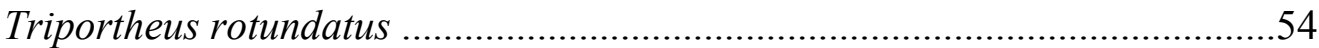

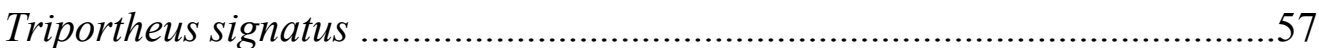

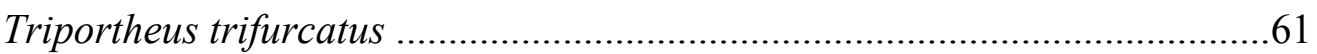


Triportheus venezuelensis

Triportheus sp. n. .66

Chave de Identificação para as espécies de Triportheus 74

Parte 2 - Estudo da musculatura cefálica das espécies de Triportheus

Introdução 82

Objetivos .84

Materiais e Métodos .85

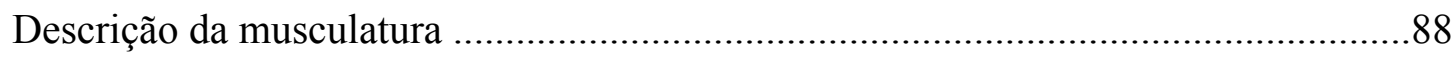

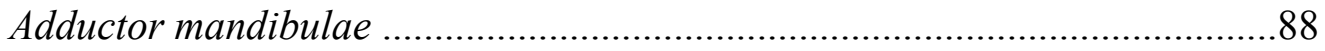

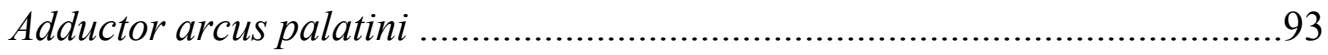

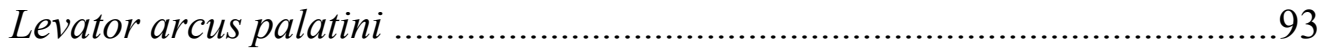



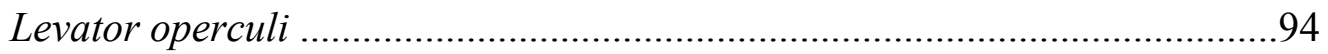

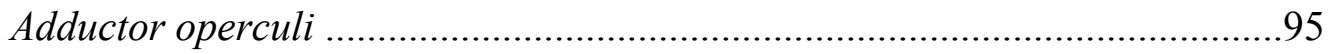

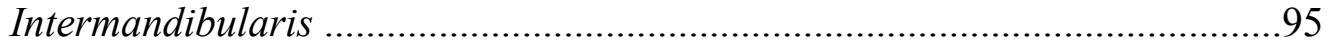

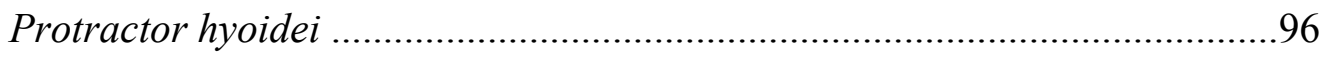



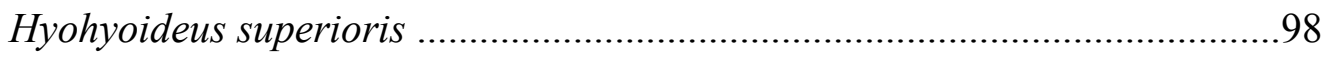

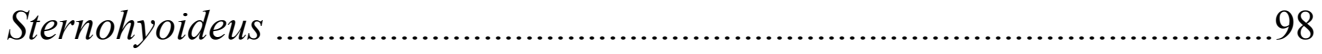

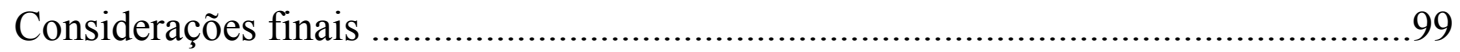

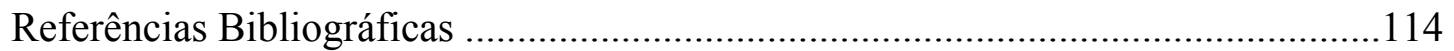

Apêndice 1: Diferenças entre os trabalhos de Portugal (1990) e Malabarba (2004) acerca das espécies de Triportheus reconhecidas. 120 


\section{LISTA DE FIGURAS}

1: Triportheus albus, MZUSP 75710, 108,0 mm CP, Brasil, Amazonas, Lago Janauari, Primeira Olaria

2: Distribuição geográfica de Triportheus albus. Círculos azuis indicam localidades fornecidas por Malabarba (2004: fig. 12). Círculos vermelhos representam lotes examinados no presente estudo. Seta indica localidade tipo. 10

3: Triportheus angulatus, MZUSP 30510, 67,1 mm CP, Brasil, Rondônia, Rio Madeira, Calama, paraná do Caraparu. .16

4: Distribuição geográfica de Triportheus angulatus. Círculos azuis indicam localidades fornecidas por Malabarba (2004: fig. 2). Círculos vermelhos representam lotes examinados no presente estudo. Seta indica localidade tipo. 17

5: Triportheus auritus, MZUSP 6669, 150,0 mm CP, Brasil, Amazonas, Rio Negro, arredores de Manaus. 26

6: Distribuição geográfica de Triportheus auritus. Círculos azuis indicam localidades fornecidas por Malabarba (2004: fig. 8). Círculos vermelhos representam lotes examinados no presente estudo. Seta indica localidade tipo. .27

7: Triportheus brachipomus, MZUSP 104764, 99,0 mm CP, Brasil, Amapá, Laranjal do Jari, Igarapé Três bocas, afl. do Pacanari, a jusante da cachoeira Santo Antônio..30

8: Distribuição geográfica de Triportheus brachipomus. Círculos azuis indicam localidades fornecidas por Malabarba (2004: fig. 2). Círculos vermelhos representam lotes examinados no presente estudo. Seta indica localidade tipo. .31

9: Triportheus culter, MZUSP 26717, 139,1 mm CP, Peru, Ucayali, Rio Ucayali, Pucallpa. .33 
10: Distribuição geográfica de Triportheus culter. Círculos azuis indicam localidades fornecidas por Malabarba (2004: fig. 12). Círculos vermelhos representam lotes examinados no presente estudo. Seta indica localidade tipo.

11: Triportheus curtus, MZUSP 26291, 69,6 mm CP, Peru Ucayali, Cashibococha, Pucallpa. 35

12: Distribuição geográfica de Triportheus curtus. Círculos azuis indicam localidades fornecidas por Malabarba (2004: fig. 8). Círculos vermelhos representam lotes examinados no presente estudo. Setas indicam localidades dos sintipos. 36

13: Triportheus guentheri, MZUSP 19656, 112,7 mm CP, Brasil, Minas Gerais, Represa de Três Marias, rio São Francisco. 39

14: Distribuição geográfica de Triportheus guentheri. Círculos azuis indicam localidades fornecidas por Malabarba (2004: fig. 8). Círculos vermelhos representam lotes examinados no presente estudo. Setas indicam localidades dos sintipos. 40

15: Triportheus magdalenae, MZUSP 36688, 141,1 mm CP, Colômbia, Cundinamarca, Cienaga La Bija, rio Magdalena perto de San Pablo. .42

16: Distribuição geográfica de Triportheus magdalenae. Círculos azuis indicam localidades fornecidas por Malabarba (2004: fig. 23). Círculos vermelhos representam lotes examinados no presente estudo. Seta indica localidade tipo. .43

17: Triportheus nematurus, MZUSP 54149, 74,8 mm CP, Paraguai, Alto Paraguay, Riacho Vaqueiro ca. 2000m acima de Fuerte Olympo na base de Cerro Barrero. .....44

18: Distribuição geográfica de Triportheus nematurus. Círculos azuis indicam localidades fornecidas por Malabarba (2004: fig.12). Círculos vermelhos representam lotes examinados no presente estudo. Seta indica localidade tipo. 45

19: Triportheus orinocensis, MZUSP 103059, 108,2 mm CP, Venezuela, Codejes, Caño de Agua, entre las poblaciones de Laguna y el Amparo. 
20: Distribuição geográfica de Triportheus orinocensis. Círculos azuis indicam localidades fornecidas por Malabarba (2004: fig. 23). Círculos vermelhos representam lotes examinados no presente estudo. Seta indica localidade tipo. .48

21: Triportheus pantanensis, MZUSP 54145, 66,7 mm CP, Paraguai, Alto Paraguay, Rio Paraguay, em frente a zona militar, na fronteira com o Brasil. 49

22: Distribuição geográfica de Triportheus pantanensis. Círculos azuis indicam localidades fornecidas por Malabarba (2004: fig. 23). Círculos vermelhos representam lotes examinados no presente estudo. Seta indica localidade tipo. .50

23: Triportheus pictus, MZUSP 30513, 75,2 mm CP, Brasil, Amazonas, Rio Tefé. .52

24: Distribuição geográfica de Triportheus pictus. Círculos azuis indicam localidades fornecidas por Malabarba (2004: fig. 4). Círculo vermelho representa o lote examinado no presente estudo. Seta indica localidade tipo. .53

25: Triportheus rotundatus, MZUSP 6876, 96,9 mm CP, Brasil, Amazonas, Lago Janauari. .54

26: Distribuição geográfica de Triportheus rotundatus. Círculos azuis indicam localidades fornecidas por Malabarba (2004: fig. 4). Círculos vermelhos representam lotes examinados no presente estudo. Seta indica localidade tipo. .55

27: Triportheus signatus, MZUSP 98720, 83,1 mm CP, Brasil, Piauí, Lagoa Azul, localidade de Ibiraba, próximo da fazenda Lagoa Azul, na estrada de Curimata.

28: Distribuição geográfica de Triportheus signatus. Círculos azuis indicam localidades fornecidas por Malabarba (2004: fig. 4). Círculos vermelhos representam lotes examinados no presente estudo. Seta indica localidade tipo. .59 
29: Triportheus trifurcatus, MZUSP 52122, 93,9 mm CP, Brasil, Tocantins, Rio Água Fria, fazenda Praia Alta 2, estrada Araguaçu/Barreira do Piqui, $27 \mathrm{~km}$ ao norte de Araguaçu.

30: Distribuição geográfica de Triportheus trifurcatus. Círculos azuis indicam localidades fornecidas por Malabarba (2004: fig. 4). Círculos vermelhos representam lotes examinados no presente estudo. .62

31: Triportheus venezuelensis, MZUSP 103068, 85,2 mm CP, Venezuela, Barinas, Laguna em Playa del Caño Fistolito. .64

32: Distribuição geográfica de Triportheus venezuelensis. Círculos azuis indicam localidades fornecidas por Malabarba (2004: fig. 12). Círculos vermelhos representam lotes examinados no presente estudo. Seta indica localidade tipo. .65

33: Triportheus sp. n., Holótipo, MZUSP 89739, 131,3 mm CP, Brasil, Mato Grosso, Rio Culuene, na cachoeira (área de futura PCH Paranatinga II). .66

34: Vista ventral do pré-maxilar de Triportheus sp. n. Setas vermelhas indicam os três dentes tricuspidados da fileira mediana. Barra $=1 \mathrm{~mm}$.

35: Distribuição geográfica de Triportheus sp. $n$. Seta indica localidade do holótipo.

36: Morfotipos de nadadeiras caudais encontradas nas espécies de Triportheus. A: T. magdalenae, MZUSP 36688, 141,1 mm CP. B: T. orinocensis, MZUSP 103059, 108,2 mm CP. C: T. rotundatus, MZUSP 6876, 96,9 mm CP. D: T. sp. n., MNRJ 35225, 83,1 mm CP. E: T. pictus, MZUSP 30513, 75,2 mm CP. .78

37: Detalhe da região do corpo ventral à nadadeira peitoral de A: Triportheus albus, MZUSP 75710, 86,8 mm CP,. B: T. pantanensis, MZUSP 54147, 76,5 mm CP, e C: T. guentheri, MZUSP 19656, 106,53 mm CP. Vista lateral esquerda. NP = nadadeira peitoral. Os exemplares foram corados com alizarina para facilitar a visualização das escamas. Setas indicam a(s) escama(s) entre a base do raio mais interno da nadadeira 
peitoral e a margem ventral da quilha, desconsiderando a escama mais ventral.

38: Detalhe da região lateral do corpo na altura da nadadeira dorsal de A: Triportheus curtus, MZUSP 26291, 54,0 mm CP e, B: T. rotundatus, MZUSP 5937, 64,0 mm CP. Vista lateral esquerda. LL $=$ Linha lateral. Os exemplares foram corados com alizarina para facilitar a visualização das escamas. Números indicam fileiras de escamas entre a linha lateral e a base do primeiro raio da nadadeira dorsal.

39: Detalhe do ramo inferior do primeiro arco branquial de A: Triportheus. venezuelensis, MZUSP 103068, 94,2 mm CP e. B: T. angulatus, MZUSP 5908, 94,6 mm CP. Vista lateral esquerda. $\mathrm{RB}=$ Rastros Branquiais. $\mathrm{FB}=$ Filamentos branquiais. Seta aponta para a região entre as bases dos rastros branquiais .80

40: Detalhe da região opercular de A: Triportheus pantanensis, MZUSP 54147, 74,4 mm CP e B: T. rotundatus, MZUSP 6876, 81,2 mm CP. Vista lateral esquerda. Linha verde indica vertical que passa pela margem posterior do subopérculo. $\mathrm{NP}=$ Nadadeira peitoral; $\mathrm{OP}=$ Opérculo; $\mathrm{PCL}=$ Pós-cleitro; SOP $=$ Subopérculo. Os exemplares foram corados com alizarina para facilitar a visualização dos ossos.

41: Desenho esquemático da vista lateral esquerda da cabeça de Triportheus albus (MZUSP 75710, 90,4 mm CP). Globo ocular e série infraorbital removidos. Seta indica divisão entre as seções A1 e A2 do adductor mandibulae. Essa divisão é meramente ilustrativa, não sendo visível no exemplar em vista lateral. 101

42: Desenho esquemático da vista lateral esquerda da cabeça de Triportheus albus (MZUSP 75710, 90,4 mm CP). Série infraorbital, opérculo, subopérculo, dilatator operculi, levator operculi, membrana primordial, ligamento primordial e parte da seção A1 do adductor mandibulae removidos. Tracejado indica fossa dilatatoris operculi. .102 
43: Desenho esquemático da vista medial da hemimandibula esquerda de Triportheus pantanensis (MZUSP 54147, 76,5 mm CP). Imagem invertida horizontalmente. ...103

44: Detalhe da cabeça, na região posterior à órbita, em vista lateral direita, de Triportheus angulatus. A:MZUSP 5908, 94,6 mm CP, e B: T. angulatus, MZUSP 75726, 128,4 mm CP,. Opérculo, dilatator operculi, levator operculi e adductor operculi removidos. Seta indica o ponto de origem da seção A2 do adductor mandibulae. 104

45: Detalhe da região orbital de Triportheus sp. n. (MZUSP 89739, 96,2 mm CP). Vista lateral esquerda. Série infraorbital, Adductor mandibulae, opérculo, préopérculo, dilatator operculi e levator operculi removidos. Tracejado indica a fossa dilatatoris operculi. Acima é uma fotografia e abaixo é um desenho esquemático da mesma região. Nem todas as estruturas foram representadas no desenho. 105

46: Detalhe da região orbital de Triportheus albus (MZUSP 75710, 90,4 mm CP)). Vista lateral esquerda. Série infraorbital, Adductor mandibulae, opérculo, préopérculo, dilatator operculi e levator operculi removidos. Tracejado indica a fossa dilatatoris operculi. Acima é uma fotografia e abaixo é um desenho esquemático da mesma região. Nem todas as estruturas foram representadas no desenho. 106

47: Desenho esquemático da vista medial do opérculo esquerdo de Triportheus albus (MZUSP 75710, 90,4 $\mathrm{mm} \quad \mathrm{CP}$ ). Imagem invertida horizontalmente.

48: Detalhe da região dorsal do opérculo de Triportheus albus (MZUSP 75710, 90,4 mm CP). Vista lateral esquerda. Acima é uma fotografia e abaixo é um desenho esquemático da mesma região. 108

49: Detalhe da região dorsal do opérculo de Triportheus signatus (MZUSP 37221, 147,2 mm CP). Vista lateral esquerda. Acima é uma fotografia e abaixo é um desenho esquemático da mesma região. 109 
50: Detalhe da região anterior do corpo de Triportheus albus (MZUSP 75710, 86,8 mm CP). Vista ventral. Acima é uma fotografia e abaixo é um desenho esquemático da mesma região.

51: Detalhe da hemimandibula esquerda de Triportheus brachipomus (MZUSP 4833, $112,5 \mathrm{~mm} \mathrm{CP})$. Vista medial. Imagem invertida horizontalmente.

52: Detalhe da região anterior do corpo de Triportheus sp. n. (MZUSP 89739, 96,2 $\mathrm{mm}$ CP). Vista ventral. Protractor hyoidei removido.

53: Detalhe da região ventral do corpo, na altura dos arcos branquiais, de Triportheus brachipomus (MZUSP 4833, 112,5 mm CP). Vista lateral esquerda. Suspensório, raios branquiostégios e arcos branquiais removidos. Acima é uma fotografia e abaixo é um desenho esquemático da mesma região. 113

\section{LISTA DE TABELAS}

1: Dados morfométricos de Triportheus angulatus. Comprimento padrão expresso em milímetros $(\mathrm{mm})$ e demais valores expressos em porcentagens $(\%)$ em relação ao comprimento padrão (medidas de estruturas no corpo) ou o comprimento da cabeça (medidas de estruturas na cabeça).

2: Dados morfométricos de Triportheus rotundatus. Comprimento padrão expresso em milímetros $(\mathrm{mm})$ e demais valores expressos em porcentagens $(\%)$ em relação ao comprimento padrão (medidas de estruturas no corpo) ou o comprimento da cabeça (medidas de estruturas na cabeça).

3: Dados merísticos de Triportheus angulatus e Triportheus rotundatus, juntamente com os dados para as mesmas espécies apresentados por Malabarba (2004). .20

4: Distribuição do número de escamas perfuradas na linha lateral nos exemplares de Triportheus angulatus e T. rotundatus examinados no presente estudo. .20 
5: Distribuição do número de rastros no ramo inferior do primeiro arco branquial nos exemplares de Triportheus angulatus e T. rotundatus examinados no presente estudo.

6: Dados morfométricos dos Triportheus de lotes provenientes da Ilha de Marajó, com o comprimento padrão expresso em milímetros $(\mathrm{mm})$ e os demais valores expressos em porcentagens (\%) em relação ao comprimento padrão ou o comprimento da cabeça. .37

7: Dados morfométricos dos Triportheus do alto Amazonas examinados no presente estudo, com o comprimento padrão expresso em milímetros ( $\mathrm{mm})$ e os demais valores expressos em porcentagens (\%) em relação ao comprimento padrão ou o comprimento da cabeça. 37

8: Dados merísticos de Triportheus do Alto Amazonas e Triportheus da Ilha de Marajó, juntamente com os dados de T. curtus e T. rotundatus apresentados por Malabarba (2004). .38

9: Dados morfométricos de Triportheus sp. n.. Comprimento padrão expresso em milímetros (mm) e demais valores como porcentagens (\%) em relação ao comprimento padrão (medidas de estruturas no corpo) ou o comprimento da cabeça (medidas de estruturas na cabeça). . .70 
PARTE 1

\section{Aspectos taxonômicos e atualização da informação sobre a distribuição geográfica das espécies de Triportheus Cope, 1872 (Teleostei, Characiformes, Characidae)}

\section{INTRODUÇÃO}

O gênero Triportheus, Cope 1872 abriga atualmente 16 espécies de peixes consideradas válidas (Malabarba, 2004:167) e conhecidas popularmente como "sardinha comum", “sardinha papuda" e "sardinha comprida" (Ferreira et al., 1998: 94-96). São facilmente reconhecidos pela presença de nadadeiras peitorais bem desenvolvidas, uma quilha peitoral na região anteroventral do corpo formada pelos ossos coracoides expandidos, corpo comprimido lateralmente e a linha lateral com uma inclinação posteroventral acentuada na região do corpo próxima da nadadeira peitoral. A maioria das espécies de Triportheus (11: T. albus Cope, T. angulatus (Spix \& Agassiz), T. auritus (Valenciennes, in Cuvier \& Valenciennes), T. brachipomus (Valenciennes, in Cuvier \& Valenciennes), T. culter (Cope), T. curtus (Garman), T. orinocensis Malabarba, T. pictus (Garman), T. rotundatus (Jardine in Shomburgk), T. trifurcatus (Castelnau) e T. venezuelensis Malabarba) ocorrem nas bacias Amazônica, do Araguaia-Tocantins e do Orinoco e nos rios costeiros entre os rios Demerara e Araguari. Duas espécies (T. pantanensis Malabarba e T. nematurus (Kner)) ocorrem na bacia do Paraná-Paraguai, uma no Rio São Francisco (T. guentheri (Garman)), uma nos rios costeiros do Nordeste brasileiro (T. signatus (Garman)) e uma na bacia do rio 
Magdalena (T. magdalenae (Steindachner)) sendo a única espécie transandina do gênero.

São peixes de pequeno a médio porte, geralmente não ultrapassando $30 \mathrm{~cm}$ de comprimento. Ocorrem em diversos ambientes, podendo até ser encontrados em águas com baixa concentração de oxigênio (Yamamoto et al., 2004:654), e são modestamente utilizados como alimento, principalmente na região amazônica, tanto na pesca comercial quanto na de subsistência das comunidades tradicionais (Ferreira et al.,1998:94-96; Amaral, 2005:137; Doria \& Queiroz, 2008:100). As espécies possuem uma dieta onívora, incluindo frutos, sementes e invertebrados (Ferreira et al., 1998:94-96)

O trabalho mais recente e completo de revisão taxonômica do gênero Triportheus é o de Malabarba (2004), no qual são redescritas 13 espécies já conhecidas, descritas três novas espécies e são apresentados mapas com as informações das distribuições geográficas de cada uma delas. Para tal estudo, a autora examinou exemplares proveniente de todas as bacias de drenagens onde o gênero ocorre de diversas coleções ictiológicas, com ênfase principalmente em material proveniente do alto Amazonas, do Orinoco e do Essequibo. A autora examinou ainda exemplares tipo da maioria das espécies nominais, resolvendo, assim diversos problemas taxonômicos que não haviam sido solucionados num trabalho anterior de revisão do gênero Triportheus, porém não publicado, realizado por Portugal (1990). O autor baseou seu estudo principalmente em material depositado no MZUSP, coleção que apesar de incluir representantes de algumas bacias hidrográficas da América do Sul fora dos limites do território brasileiro, caracteriza-se por possuir uma ampla representatividade dos rios da região Amazônica brasileira. Por outro lado, Malabarba (2004) examinou muito pouco material proveniente da coleção do MZUSP (apensa 25 
dos quase 400 lotes de Triportheus depositados até então) e acarretando na presença de grandes hiatos na distribuição geográfica de várias das espécies de Triportheus nos rios da bacia Amazônica. No entanto, com relação ao material tipo, Portugal (1990) não teve acesso direto a todos os exemplares tipos disponíveis das espécies nominais, tendo examinado muitos deles apenas por fotografias e dados merísticos e morfométricos enviados a ele. Além disso, diferenças de interpretação entre os estudos de Malabarba (2004) e Portugal (1990) acerca das descrições originais de espécies nominais em que os exemplares tipo são dados como perdidos, levaram a diferenças na resolução de alguns problemas taxonômicos e das espécies consideradas válidas para cada autor. Por exemplo Portugal (1990) considerou quatro espécies nominais como species inquirenda devido à imprecisão, na visão do autor, no reconhecimento da espécie apenas pelas informações disponíveis nas descrições originais (Triportheus angulatus, Chalcinus muelleri, Chalcinus knerii e Chalcinus auritus). Por outro lado, Malabarba (2004), considera T. angulatus e Chalcinus auritus (= T. auritus) como válidas, e Chalcinus muelleri e C. knerii foram considerados sinônimo júnior de uma outra espécie ou por obtenção de material tipo não examinado por Portugal (1990) ou com base em dados presentes nas descrições originais.

O exame de material não incluído no estudo de Malabarba (2004) das drenagens da bacia amazônica mostrou que T. angulatus e T. rotundatus espécies ocorrem em simpatria em diversos rios amazônicos e apresentam uma sobreposição nas amplitudes no número de escamas perfuradas na linha lateral e de rastros no ramo inferior do primeiro arco branquial, sendo esses valores utilizados para diferenciar as duas espécies (Malabarba, 2004:170). Outro aspecto está relacionado com a espécie T. curtus. A localidade tipo desta espécie é na ilha de Marajó e apenas o único lote de 
material não tipo examinado por Malabarba (2004:192) é proveniente do Alto rio Purus. Esse enorme hiato na distribuição geográfica de T. curtus pode ser decorrente da falta de exame de material proveniente dos rios da bacia amazônica ou ainda indicar a existência de um problema taxonômico.

Portugal (1990:115) indica a da existência de uma possível espécie nova no rio Xingu, baseado no exame de 9 exemplares provenientes da Cachoeira do Espelho, município de Altamira. O aumento recente do número de exemplares oriundos dessa drenagem em diversas coleções, em particular as do MZUSP e do Museu Nacional do Rio de Janeiro (MNRJ), permite uma avaliação mais detalhada dessa questão.

Um último aspecto presente no trabalho da autora são diversas chaves de identificação regionais de Triportheus. Segundo Malabarba (2004:169) isso facilitaria a identificação das espécies devido a pouca variação morfológica intragenérica e a restrição da maioria das espécies a apenas uma drenagem sul-americana. No entanto, o exame preliminar do material de Triportheus disponível mostrou que $T$. brachipomus e T. signatus estariam presentes em bacias hidrográficas não registradas pelo trabalho de Malabarba (2004). Em vista disso, optou-se por fazer uma chave de identificação geral incluindo todas as espécies do gênero.

\section{OBJETIVOS}

Este trabalho tem como objetivos:

1- Atualizar as informações sobre a distribuição geográfica das espécies de Triportheus, com ênfase nos rios da Amazônia brasileira.

2- Avaliar a amplitude de variação dos caracteres propostos como diagnósticos para as espécies, principalmente $T$. angulatus e $T$. rotundatus. 
3- Investigar a ocorrência de um possível problema taxonômico envolvendo $T$. curtus

4- Investigar a existência de uma possível espécie nova na bacia do Rio Xingu.

\section{MATERIAIS E MÉTODOS}

A atualização da informação sobre a distribuição geográfica das espécies de Triportheus foi feita com base no exame de 517 lotes depositados no Museu de Zoologia da Universidade de São Paulo (MZUSP), 40 depositados no Instituto de Pesquisa da Amazônia (INPA), 7 no Museu Nacional do Rio de Janeiro (MNRJ), e 9 no Museu Paraense Emílio Goeldi (MPEG), num total de 573 lotes. O foco do presente trabalho em exemplares da Amazônia brasileira se deve ao fato de que os hiatos nas informações das distribuições geográficas das espécies serem principalmente na região amazônica brasileira, região onde ocorrem a maioria das espécies do gênero.

As coordenadas geográficas, quando não foram fornecidas pelos coletores, foram determinadas com o auxílio de mapas diversos, do programa Google Earth $^{\circledR} \mathrm{e}$ do site do Geographic Names Server (http://earth-info.nga.mil/gns/html/index.html). Mesmo quando as coordenadas geográficas eram informadas no lote examinado, elas eram confirmadas em mapas com o intuito de evitar eventuais discrepâncias com relação a localidade de coleta do lote. A partir desses dados, foram construídos mapas de distribuição utilizando em conjunto os programas Google Earth ${ }^{\circledR}$ e o Quantum GIS $^{\circledR}$, com o auxílio do tutorial presente no website Wikipeixes (http://www.wikipeixes.com.br/tutoriais). Devido a falta de informações completas 
sobre localidades de coleta de alguns lotes estas não foram incluídas nos mapas de distribuição. Porém, tomou-se o cuidado de sempre se verificar, com base na informação que havia, se a localidade de coleta estava dentro dos limites de abrangência geográfica da espécie. A informação das localidades de ocorrência de cada espécie fornecidas por Malabarba (2004), foram incluídas nos mapas preparados no presente trabalho, com cores distintas para facilitar a comparação das informações entre os dois trabalhos. Os dados das localidades fornecidos no trabalho de Malabarba (2004) também foram conferidos.

Dados merísticos e morfométricos foram tomados de acordo com Fink \& Weitzman (1974) com as modificações propostas por Malabarba (2004:168), exceto a contagem de séries de escamas no pedúnculo caudal, a qual foi considerada a série completa ao redor do pedúnculo e não de apenas de um lado do corpo.

As medidas tomadas foram as seguintes: comprimento padrão, comprimento da cabeça, distância pré-anal, distância pré-dorsal, distância pré-pélvica, distância prépeitoral, comprimento da base da nadadeira anal, comprimento da base da nadadeira dorsal, comprimento do pedúnculo caudal, altura do pedúnculo caudal, altura do corpo na origem da nadadeira dorsal, maior altura do corpo, comprimento da nadadeira anal, comprimento da nadadeira dorsal, comprimento da nadadeira pélvica, comprimento da nadadeira peitoral, distância entre a base do último raio da nadadeira dorsal e a base da nadadeira adiposa, comprimento do focinho, comprimento da maxila superior, diâmetro horizontal do olho, menor distância interorbital e comprimento da região pós-orbital da cabeça. Todas essas medidas foram expressas como porcentagens do comprimento padrão, exceto as cinco últimas (que incluem as medidas de estruturas na cabeça) que foram expressas como porcentagens do comprimento da cabeça. 
Os dados merísticos tomados foram os seguintes: número de escamas perfuradas na linha lateral, número de escamas entre a base do último raio da nadadeira peitoral e a margem ventral da quilha peitoral, número de escamas entre a linha lateral e a origem da nadadeira dorsal, número de escamas entre a linha lateral e a margem ventral do corpo na vertical que passa pela origem da nadadeira pélvica, número de raios ramificados da nadadeira anal, número de rastros no ramo inferior do primeiro arco branquial, escamas ao redor do pedúnculo caudal e fileiras de dentes no pré-maxilar. O número de raios das nadadeiras peitoral, pélvica e dorsal e o número de dentes do pré-maxilar, dentário e maxilar foram incluídos apenas para os exemplares da espécie nova descrita uma vez que estas características não se mostraram diagnósticas para nenhuma espécie no estudo de Malabarba, 2004.

Medidas foram feitas com o auxílio de um paquímetro digital Mitutoyo, modelo CD-8"C com precisão de $0,1 \mathrm{~mm}$. Os exemplares foram examinados com o auxílio de um Esteromicroscópio Leica MZ 12,5. Os registros fotográficos foram feitas com uma câmera digital Leica DC 300 acoplada ao estereomicroscópio e uma câmera digital Canon EOS Rebel XTI.

Nas listas de materiais examinados de cada espécie consta a sigla da instituição onde o material está depositado (o asterisco indica um lote também examinado por Malabarba (2004)), número de registro, número de exemplares do lote, a amplitude de comprimento padrão em milímetros e dados de localidade, ordenados por país e estado (ou departamento), ambos em ordem alfabética. Lotes cujas coordenadas geográficas não foram determinadas estão incluídos na lista de material examinado, porém não nos mapas de distribuição geográfica das espécies.

Apesar do trabalho de Portugal (1990) não estar publicado, ao comparar seus resultados com os de Malabarba (2004), surgem diversas dúvidas acerca da 
taxonomia e da distribuição das espécies do gênero, como mencionado anteriormente. Uma comparação entre os dois trabalhos é apresentada no apêndice 1, juntamente com uma explicaçãoo das diversas diferenças encontradas acerca das espécies de Triportheus reconhecidas como válidas.

\section{RESULTADOS E DISCUSSÃO}

A seguir são apresentadas informações sobre a distribuição geográfica de cada espécie válida de Triportheus, em ordem alfabética, juntamente com um mapa e alguns comentários acerca da espécie Para informações adicionais de cada espécie, ver Apêndice 1. 


\section{Triportheus albus Cope, 1872}

(Figura 1)

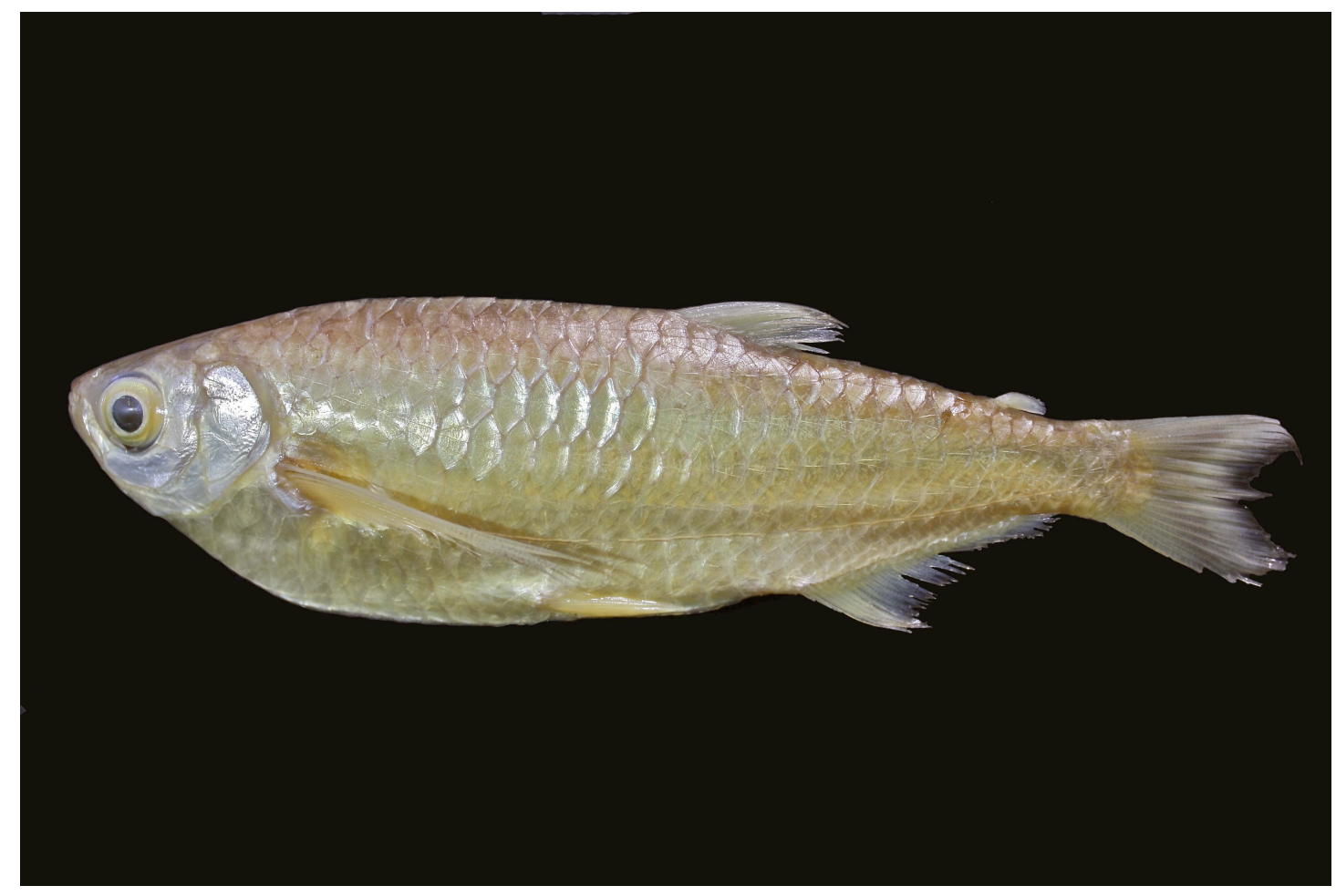

Figura 1: Triportheus albus, MZUSP 75710, 108,0 mm CP, Brasil, Amazonas, Lago Janauari, Primeira Olaria.

Essa espécie está amplamente distribuída nos rios da Bacia Amazônica, incluindo toda a extensão do rio Amazonas e Solimões, além das drenagens dos rios Xingu, Tapajós, Trombetas, Madeira, Negro, Purus, Branco, Napo, Yasuni, Ucayali e Ambiyacu, e em rios da Bacia do Tocantins-Araguaia (figura 2). Localidade tipo: rio Ambiyacu, próximo de Pebas. 




Figura 2: Distribuição geográfica de Triportheus albus. Círculos azuis indicam localidades fornecidas por Malabarba (2004: fig. 12). Círculos vermelhos representam lotes examinados no presente estudo. Seta indica localidade tipo.

Comentários sobre Triportheus albus

Triportheus albus, a espécie tipo do gênero, foi descrita por Malabarba (2004: fig. 12) como ocorrendo nos rios Araguaia, Tapajós, Amazonas, Negro, Itenez (= rio Guaporé), Napo, Yasuni, Ambiyacu e Ucayali. No presente estudo foram registrados diversos pontos de ocorrência da espécie ao longo dos rios Solimões e Amazonas, e no baixo rio Tocantins. Além disso, T. albus também ocorre nos rios Acre, Purus, Tiquié, Aripuanã, Trombetas e Xingu. A adição dessas localidades à informação da distribuição geográfica da espécie mostrou que existe continuidade entre as ocorrências propostas por Malabarba (2004: fig. 12). 
Material examinado (147 lotes; 2370 exemplares; 12,6-218,9 mm CP)

BRASIL: Acre: MZUSP 49648, 2, 37,9-43,1 mm CP; Rio Acre, Seringal do Acre. MZUSP 49670, 11, 32,6-53,5 mm CP; Rio Acre, Seringal Bom Destino (1 hora acima de Porto Acre). MZUSP 50461, 2, 100,9-104,4 mm CP; Lago Calila, Rio Juruá. MZUSP 49627, 5, 25,2-43,2 mm CP; Rio Acre, Colocação do Chalé, 6 horas abaixo de Rio Branco. MZUSP 49843, 2, 102,9-115,8 mm CP; Riozinho da Rola. Amapá: MZUSP 59099, 5, 134,0-174,9 mm CP; Rio Cupixi, ponte na estrada para a Serra do Navio (canal do rio). Amazonas: MZUSP 5852*, 13; Rio Negro. MZUSP 7581, 101; Paraná do Mocambo, perto de Paritins. MZUSP 19781, 2, 69,6-76,3 mm CP; Lagoa junto ao canal do lago Janauacá, margem direita do rio Solimões. MZUSP 20398, 2, 77,1-79,2 mm CP; Lago Janauacá e arredores, Rio Solimões. MZUSP 30508, 1; Rio Negro, Anavilhanas, lago do Prato. MZUSP 49517, 1, 102,8 mm CP; Rio Acre. MZUSP 49553, 1; Rio Acre, junto ao lago Lua Nova. MZUSP 49604, 149, 20,8-56,5 mm CP; Rio Acre, Seringal Floresta, 10 min. acima de Boca do Acre. MZUSP 49692, 16, 23,7-47,1 mm CP; Rio Acre, 1 hora abaixo do lago Lua Nova. MZUSP 49709, 26, 16,3-48,1 mm CP; Rio Purus, Boca do Acre. MZUSP 56147, 31, 23,9-48,4 mm CP; Rio Solimões, 34,3 km abaixo Juruá. MZUSP 58159, 1, 134,7 mm CP; Rio Amazonas, 1,85 km abaixo da boca do lago Puraquequara. MZUSP 67925, 3, 117,9129,9 mm CP; Lago Pirapora, margem esquerda do Rio Acre, AM 16 km município do Porto Acre. MZUSP 75695, 250, 17,1-42,0 mm CP; Lago Terra Preta, Janauari. MZUSP 75696, 14, 56,7-58,0 mm CP; Entrada de Janauari, entre o Furo de Paracuuba e Lagoa Terra Preta. MZUSP 75697, 3, 107,3-116,8 mm CP; Camaleão, Ilha de Marchantaria. MZUSP 75698, 6; Ressaca da Ilha de Marchantaria. MZUSP 75699, 3, 88,5-89,9 mm CP; Paraná da Ilha de Marchantaria. MZUSP 75700, 21, 43,9-54,6 mm CP; Lago Terra Preta, Janauari. MZUSP 75701, 11, 72,2-78,8 mm CP; 
Paraná da Ilha da Marchantaria. MZUSP 75702, 12; Camaleão, Ilha de Marchantaria. MZUSP 75703, 6, 73,6-97,3 mm CP; Ressaca da Ilha de Marchantaria. MZUSP 75704, 8, 84,4-94,8 mm CP; Ressaca da Ilha de Marchantaria. MZUSP 75706, 5, 82,7-96,0 mm CP; Ressaca da Ilha de Marchantaria. MZUSP 75707, 45, 75,5-95,1 mm CP; Paraná da Ilha de Marchantaria. MZUSP 75708, 31, 69,2-110,5 mm CP; Lago Terra Preta, Janauari. MZUSP 75709, 8; Ressaca da Ilha de Marchantaria. MZUSP 75710, 14, 78,3-111,9 mm CP; Lago Janauari, primeira Olaria. MZUSP 81513, 1, 167,8 mm CP; Rio Tiquié, comunidade de Caruru, tombo da cachoeira do Caruru. MZUSP 59239, 7, 59,1-71,3 mm CP; Entrada de Janauari, perto do lago Basílio. MZUSP 77497, 2, 20,1-24,4 mm CP; Praia no Rio Solimões, na Ilha Muratu, em frente a boca do Lago Janauacá. MZUSP 77481, 6, 40,6-52,7 mm CP; Praia no Rio Solimões, na Ilha Muratu, em frente a boca do Lago Janauacá. MZUSP 6377, 6; Lago Beruri, Rio Purus. MZUSP 30511, 2, 109,5-116,2 mm CP; Rio Negro, logo abaixo de Daraá, Lago central-rapiché. MZUSP 59013, 4, 213,7-218,9 mm CP; Rio Marauiá, lago na beirado rio. MZUSP 5774, 1, 99,85 mm CP; Paraná do Urucará. MZUSP 5909, 2, 128,9-163,0 mm CP; Lago Jacaré, margem direita do rio Solimões, acima de Manacapuru. MZUSP 5997, 3; Lago Beruri, rio Purus. MZUSP 6303, 13, 92,2-105,0 mm CP, Lago Castro, boca do rio Purus. MZUSP 6877, 1, 104,5 mm CP; Lago Janauari. MZUSP 6950, 8, 16,5-26,0 mm CP; Rio Madeira, $25 \mathrm{~km}$ abaixo de Nova Olinda. MZUSP 20020, 9, 16,9-43,0 mm CP; Rio Solimões. MZUSP 20042, 3; Ilha Xibeco, rio Solimões, acima da boca do rio Jutaí. MZUSP 20058, 2; Boca do Rio Iça, Santo Antônio do Iça. MZUSP 20060, 75, 21,1-48,5 mm CP; Boca do Rio Iça, Santo Antônio do Iça. MZUSP 20072, 1; Rio Solimões, próximo da Ilha Baruruá, acima da boca do rio Jutaí. MZUSP 20110, 219, 13,7-33,3 mm CP; Rio Solimões, em frente a Jacaré, próximo de Fonte Boa. MZUSP 20111, 10; Rio Solimões, em frente a 
Jacaré, próximo de Fonte Boa. MZUSP 20131, 67, 18,0-27,8 mm CP; Rio Solimões. MZUSP 20139, 1; Rio Solimões, Caiambé, abaixo de Tefé. MZUSP 20143, 2, 24,526,4 mm CP; Boca do Lago Caiambé, abaixo de Tefé. MZUSP 20148, 16, 12,6-29,6 mm CP; Poça da Ilha Sorubim, rio Solimões, acima de Coari. MZUSP 20157, 102, 19,0-43,2 mm CP; Rio Solimões, Ilha Sorubim, acima de Coari. MZUSP 20158, 32 , 83,9-130,5 mm CP; Rio Solimões, Ilha Sorubim, acima de Coari. MZUSP 27313, 4; Boca do Rio Purus, Rio Solimões. MZUSP 49206, 6, 171,0-181,0 mm CP; Rio Marauiá, perto da boca, Ilha Marubim. MZUSP 49809, 1; Lago Lua Nova. MZUSP 53642, 1, 22,7 mm CP; Lago Puraquequara, Rio Amazonas, margem esquerda. MZUSP 59205, 2, 44,9-48,0 mm CP; Poça da Pedra, margem do Rio Negro, Paricatuba. MZUSP 59240, 9; Ressaca da Ilha de Marchantaria. MZUSP 59244, 11; Ressaca da Ilha de Marchantaria. MZUSP 62077, 4, 58,0-68,2 mm CP; Rio Negro, Tapera, Poça de Praia. MZUSP 63308, 7, 18,4-27,1 mm CP; Rio Negro, Anavilhanas, praia. MZUSP 63399, 1, 31,1 mm CP; Praia no Rio Solimões, na Ilha Muratu, em frente a boca do Lago Janauacá, MZUSP 74811, 1, 23, 84,0 mm CP; Maraã, água preta, margem esquerda. MZUSP 76197, 1, 41,5 mm CP; Rio Negro Anavilhamas (capinzal). MZUSP 74582, 5; Praia no Rio Solimões, na Ilha Muratu, em frente a boca do Lago Janauacá. MZUSP s/n (desdobrado de MZUSP 7582), 1, 98,6 mm CP; Paraná do Mocambo, perto de Parintins. INPA 2384, 20, 75,0-82,1 mm CP; Rio Solimões, Lago do Castanho, Janauacá. INPA 2711, 1, 168,4 mm CP; Rio Uatumã, Igarapé Samauma. INPA 5838, 18, 95,4-115,9 mm CP; Rio Solimões, Ilha da Marchantaria. INPA 24126, 1, 183,3 mm CP; Lago Mututuca, Rio Juma, Rio Aripuanã. INPA 25527, 1, 145,97 mm CP; Cachoeira Morena. INPA 13952, 1 (haviam 2 no frasco), 61,3-85,1 mm CP; Rio Solimões, Ilha da Marchantaria, baixo da enseada na área de jusante da ilha. INPA 17113, 15, 74,7-86,3 mm CP; Rio Purus, 
Furo no Lago do Sacado. INPA 19310, 2, 92,2-108,1 mm CP; Rio Japurá, margem Reserva Mamirauá. Boca do Lago Mamirauá. INPA 22319, 2, 108,8-112,9 mm CP; Catalão, lagos do Padre Madalena e Boca do Igarapé do Cuiu Goiás: MZUSP 53867, 1, 107,9 mm CP; Rio Araguaia, Lago Ressaca, Luís Alves. MZUSP 88114, 1, 128,1 mm CP; Praia do Horley. MZUSP 4833, 5; Rio Araguaia. MZUSP 19557, 2, 65,571,5 mm CP; Tocantins. Maranhão: MZUSP 4985, 8; Rio Tocantins (em poça marginal de vazante). Mato Grosso: MZUSP 30506, 1; Rio Madeira, Aripuanã, beira do rio. MZUSP 53588, 2, 109,6-120,3 mm CP; Rio Araguaia, Lago da Alvoradinha. MZUSP 53589, 2, 107,9-111,2 mm CP; Rio Araguaia, Lago do Furo da Aldeia. MZUSP 53590*, 4, 102,0- 114,4 mm CP; Rio Araguaia, Furo próximo à Aldeia Fontora. MZUSP 59019, 7, 163,1-196,1 mm CP; Rio Arinos, poço de várzea. MZUSP 19632, 1, 58,9 mm CP; Rio Araguaia. MZUSP 54292, 1, 110,4 mm CP; Rio Araguaia, São Felix do Araguaia. MZUSP 60384, 4, 85,1-86,9 mm CP; Rio Aripuanã, Porto de Balsa (rio acima), estrada que liga o distrito de Colniza a Panelas, km 18. MZUSP 62786, 2, 144,2-174,7 mm CP; Rio Teles Pires, Pesqueiro do Dentinho. MZUSP 62797, 1, 89,8 mm CP; Rio Arinos, ilha do Túlio, c. de $3 \mathrm{~km}$ acima de sua desembocadura no rio Juruena. MZUSP 54432, 1, 106,5 mm CP; Rio Araguaia. MZUSP 63047, 2, 95,1-98,3 mm CP; Rio Araguaia, Ouro Fino, 30 km a jusante de Barra do Garças. MZUSP 89907, 7; Rio Culuene, afl. Rio Xingu. Canarana/Gaúcha do Norte. MZUSP 94157, 6, 43,4-162,3 mm CP; Lagoão, Fazenda Luar do Sertão (Bacia do Rio Culuene. MZUSP 94373, 11, Lago da Miriam, margem direita do Rio Culuene. Pará: MZUSP 8521, 4, Rio Tapajós. MZUSP 19697, 4; Lago do Jacaré, Rio Trombetas. MZUSP 21898, 3; Rio Tapajós, Maloquinha, perto de Itaituba. MZUSP 34062, 312, 27,6-55,3 mm CP; Rio Xingu, poço de praia. MZUSP 34063, 2, 22,730,9 mm CP; Rio Xingu, perto da cachoeira (poço de pedral). MZUSP 34066, 2; Rio 
Xingu, poço de praia. MZUSP 34067, 21, 37,0-39,3 mm CP; Rio Tapajós, São Luis acima de Itaituba. MZUSP 47687, 9, 104,6-107,8 mm CP; Rio Xingu; MZUSP 92668, 3, 21,0-38,1 mm CP; Rio Tapajós, entre Itaituba e as cachoeiras de Pimental. MZUSP 92810, 2, 27,4-108,7 mm CP; Rio Tapajós, margem esquerda, em Itaituba. MZUSP 34065, 1, 32,2 mm CP; Rio Tapajós, Pederneiras, abaixo de Itaituba (poço de pedral). MZUSP 20506, 1, 42,8 mm CP; Rio Tocantins, Marabá. MZUSP 20629, 1, 87,6 mm CP; Rio Tocantins, entre Mocajuba e Baião. MZUSP 20631, 10, 22,7-39,4 mm CP; Lagoa marginal do Rio Tocantins, perto de Baião. MZUSP 36818, 188, 25,139,5 mm CP; Cachoeira do Espelho, rio Xingu. MZUSP 59008, 1, 159,7 mm CP; Rio Xingu, Belo Monte. MZUSP 59230, 13; Rio Tapajós, São Luis acima de Itaituba. MZUSP 73434, 3, 76,5-81,1 mm CP; Rio Itacaiunas, Caldeirão. MZUSP 97392, 1, 107,4 mm CP; Rio Jamanxim, na Prainha, próximo a Novo Progresso. Rondônia: MZUSP 30514, 3; Rio Madeira, boca do rio Machado. MZUSP 30519, 12; Rio Madeira, Calama, paraná do Caraparu. MZUSP 85216, 1, 54,8 mm CP; Rio Machado, próximo à foz. MZUSP 30520, 1; Rio Machado, Jamarizinho. MZUSP 35598, 10; Rio Madeira, Calama. MZUSP 101612, 1, 141,8 mm CP; Rio Jari, marg. dir. montante de Cachoeira Santo Antônio, montante de Porto Sabão. MZUSP 94129, 2, 85,8-87,0 mm CP. INPA 17544, 1, 123,3mm CP; Rio Jamari, canal de desvio da UHE Samuel. Roraima: MZUSP 20241, 1; Rio Branco. MZUSP MZUSP, 20253, 1, 26,5 mm CP; Rio Branco, 20km abaixo de Boiaçu. MZUSP 34071, 125, 36,1-71,8 mm CP; Rio Branco, cachoeira do Bem Querer (remanso). MZUSP 58959, 1, 27,5 mm CP; Rio Branco, cachoeira do Bem Querer (remanso). MZUSP 77338, 1, 36,4 mm CP; Igarapé do bota Panela, peto da cachoeira do Bem Querer, Caracaraí, Rio Branco. MZUSP 74117, 6; Rio Branco, Marará, Praia. Tocantins: MZUSP 83812, 3, 106,2-131,4 mm 
CP; Rio Palmeiras, a 10km da balsa pela estrada TO 485 em direção a Ponte Alta do Bom Jesus.

PERU: Loreto: MZUSP 15269, 1; Rio Samiria, Loreto. MZUSP 26505, 2; San Lorenzo, Pucallpa, Depto. de Loreto. Ucayali: MZUSP 26163, 2; Rio Ucayali, Bagazan. MZUSP 26449, 2, 65,7 mm CP; Yarinacocha, Pucallpa. MZUSP 26715, 7, 76,7-92,8 mm CP; Rio Ucayali, Pucallpa.

Triportheus angulatus (Spix \& Agassiz, 1829)

(Figura 3)

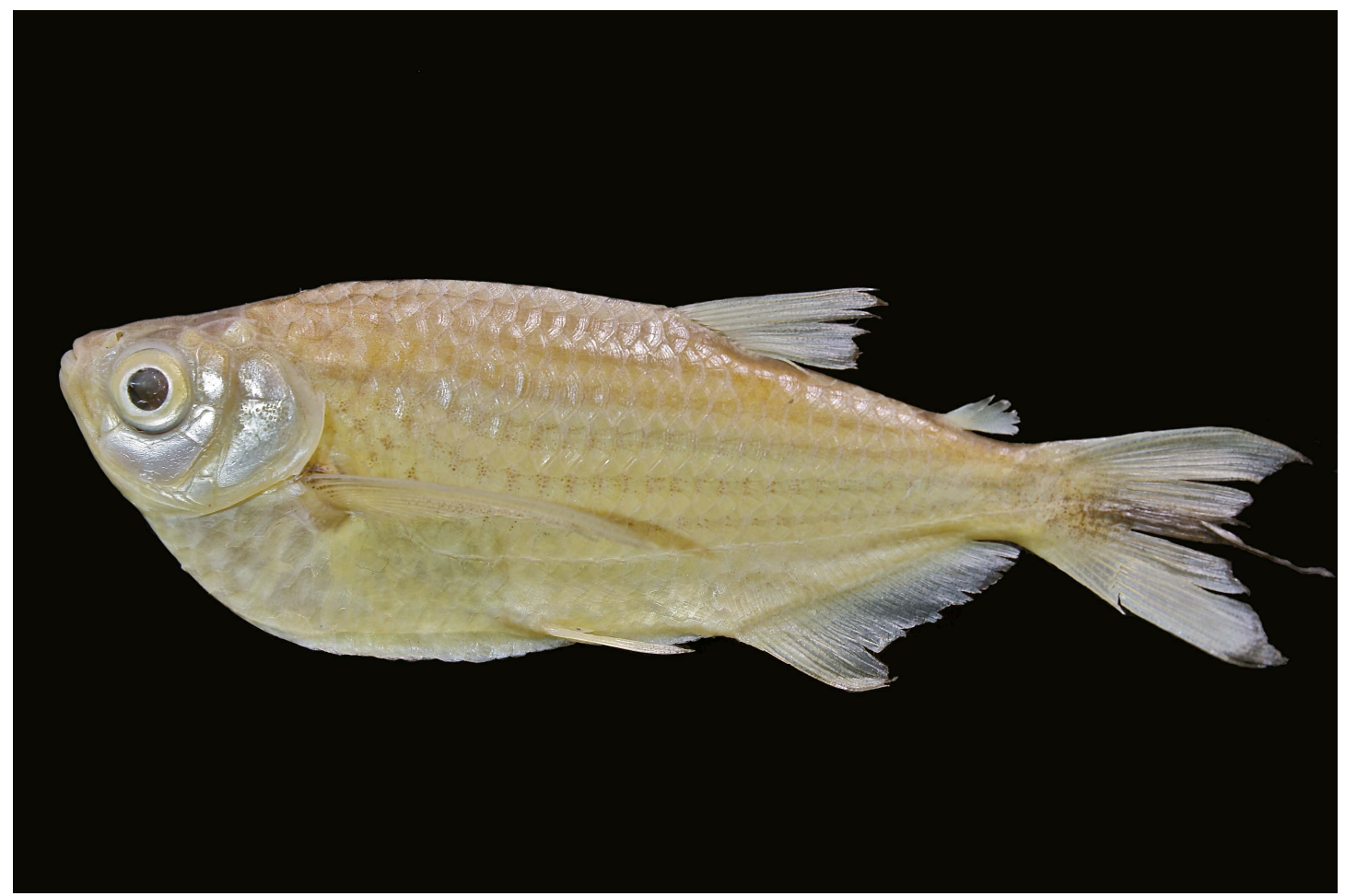

Figura 3: Triportheus angulatus, MZUSP 30510, 67,1 mm CP, Brasil, Rondônia, Rio Madeira, Calama, paraná do Caraparu.

Essa espécie está amplamente distribuída nos rios da Bacia Amazônica incluindo toda extensão dos rios Amazonas e Solimões, rios Trombetas, rio Tapajós, rio Madeira, rio Negro, rio Purus, rio Branco, rio Yasuni, rio Ucayali e rio Madre de 
Dios, e no rio Essequibo (figura 4). Na figura apresentada por Malabarba (2004: fig. 2) a localidade do neótipo está representada próxima da região de cabeceira do rio Envira (Acre). Contudo, o ponto correto deveria estar próximo à boca do rio Tefé, conforme a informação da localidade do exemplar designado pela autora como neótipo (MCP 29934, Brasil, Amazonas, rio Solimões, Ilha do Prego, Malabarba 2004:172).

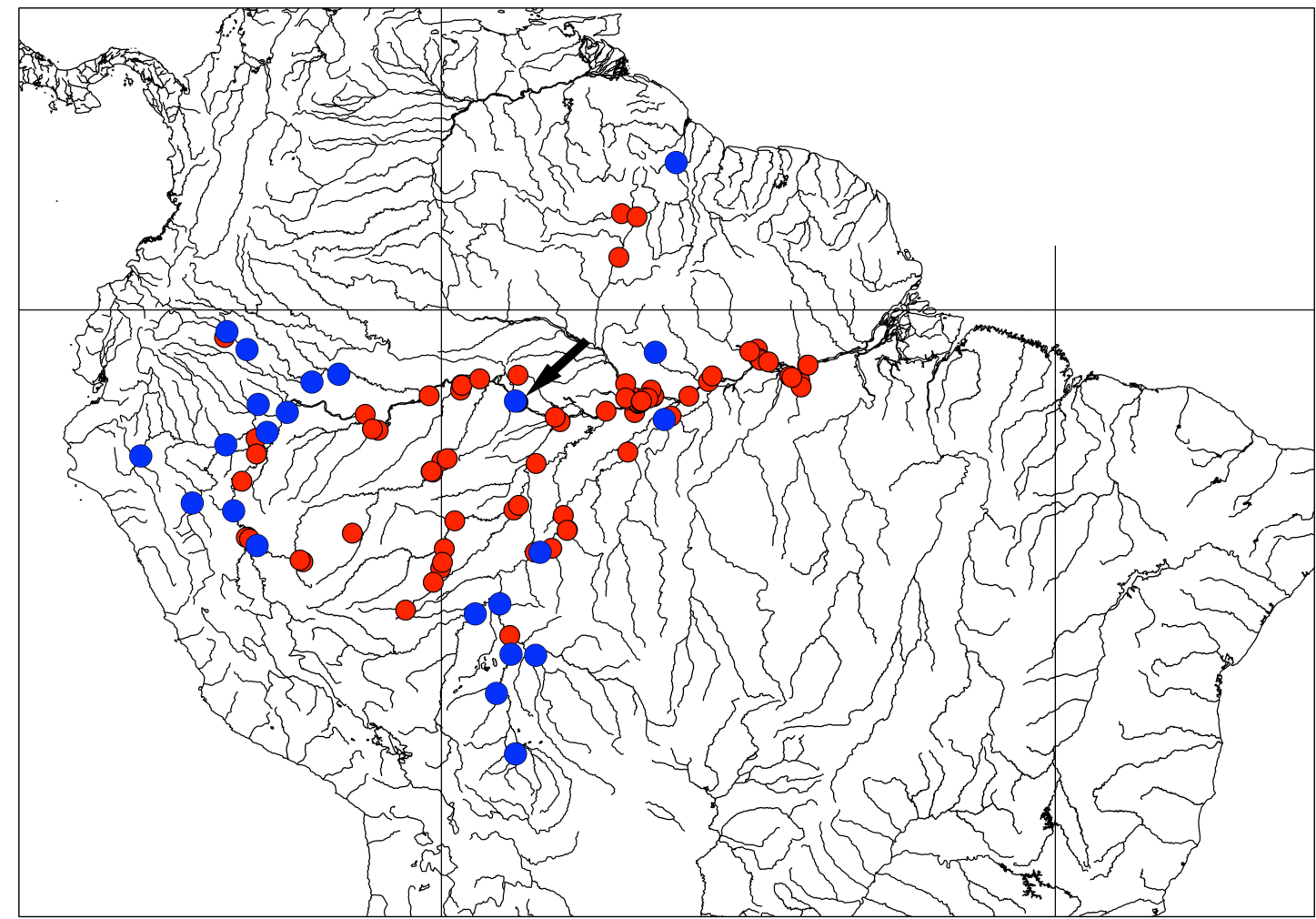

Figura 4: Distribuição geográfica de Triportheus angulatus. Círculos azuis indicam localidades fornecidas por Malabarba (2004: fig. 2). Círculos vermelhos representam lotes examinados no presente estudo. Seta indica localidade tipo.

\section{Comentários sobre Triportheus angulatus}

Triportheus angulatus é a espécie válida mais antiga do gênero, e segundo Malabarba (2004: fig. 2) ocorre nos rios Essequibo, Urubu, Madeira, Solimões, Madeira, Mamoré, Beni, Yasuni, Putumayo, Ucayali e Madre de Dios. O presente 
estudo mostrou que a espécie está mais amplamente distribuída com diversos pontos de ocorrência ao longo dos rios Solimões e Amazonas. Além disso, de T. angulatus também ocorre nos rios Juruá, Acre, Purus, Negro e Trombetas. A adição dessas localidades a informação da distribuição geográfica da espécie mostrou que existe continuidade entre as áreas de ocorrência propostas por Malabarba (2004: fig. 12).

No trabalho de Malabarba, 2004, pelo fato de Triportheus angulatus e Triportheus rotundatus apresentarem sobreposição de todos os dados morfométricos e merísticos, apesar do último ser utilizado pela autora para diagnosticar as duas espécies, e também por elas ocorrerem em simpatria, houve a necessidade de verificar se não se tratavam de uma única espécie. O acréscimo de dados de exemplares amazônicos brasileiros poderia vir a auxiliar na resolução desse problema, visto que pouco material oriundo dessa região foi examinado por Malabarba, 2004. Os dados se encontram nas tabelas 4 e 5 .

Tabela 1: Dados morfométricos de Triportheus angulatus Comprimento padrão expresso em milímetros $(\mathrm{mm})$ e demais valores expressos em porcentagens $(\%)$ em relação ao comprimento padrão (medidas do corpo) ou o comprimento da cabeça (medidas da cabeça).

\begin{tabular}{lccccc}
\hline Medida & $\mathrm{N}$ & Mínimo & Máximo & Média & DP \\
\hline Comprimento padrão & 114 & 43,5 & 160,4 & & \\
& Porcentagem do romprimento & padrão & & & \\
Comprimento da cabeça & 114 & 22,5 & 28,7 & 25,4 & 1,361 \\
Distância pré-anal & 114 & 67,0 & 76,0 & 71,7 & 1,609 \\
Distância pré-dorsal & 114 & 57,8 & 64,0 & 60,5 & 1,275 \\
Distância pré-pévica & 114 & 47,9 & 58,4 & 53,1 & 1,507 \\
Distância pré-peitoral & 114 & 23,3 & 29,2 & 26,1 & 1,304 \\
Base da nadadeira anal & 114 & 24,6 & 30,5 & 27,3 & 1,150 \\
Base da nadadeira dorsal & 114 & 9,8 & 14,6 & 11,1 & 0,709 \\
Comprimento do pedúnculo caudal & 114 & 8,4 & 11,8 & 10,2 & 0,701 \\
Altura do pedúnculo caudal & 114 & 7,5 & 10,2 & 8,9 & 0,488 \\
Altura do corpo na origem da nadadeira dorsal & 114 & 27,3 & 37,4 & 32,7 & 1,794 \\
Maior altura do corpo & 114 & 33,8 & 41,3 & 36,9 & 1,450 \\
Comprimento da nadadeira dorsal & 113 & 15,9 & 24,4 & 20,3 & 1,379 \\
Comprimento da nadadeira pélvica & 111 & 10,8 & 19,0 & 15,7 & 1,523 \\
Comprimento da nadadeira peitoral & 114 & 31,1 & 44,1 & 38,8 & 2,192 \\
Comprimento da nadadeira anal & 107 & 11,5 & 20,4 & 16,5 & 2,056 \\
Distância entre a base do último raio da nadadeira & 114 & 13,7 & 21,4 & 19,2 & 1,162 \\
dorsal e a base da nadadeira adiposa & & & & &
\end{tabular}

Porcentagens do comprimento da cabeça 
Comprimento do focinho

Comprimento da maxila superior

Diâmetro horizontal do olho

Menor distância interorbital

Comprimento da região pós-orbital da cabeça

$\begin{array}{lllll}114 & 17,0 & 27,9 & 23,4 & 2,035 \\ 114 & 32,6 & 42,9 & 36,7 & 2,041 \\ 114 & 28,2 & 39,9 & 34,0 & 2,520 \\ 114 & 31,2 & 42,7 & 36,7 & 2,385 \\ 114 & 38.8 & 51,7 & 44,4 & 2,505\end{array}$

Tabela 2: Dados morfométricos de Triportheus rotundatus. Comprimento padrão expresso em milímetros $(\mathrm{mm})$ e demais valores expressos em porcentagens $(\%)$ em relação ao comprimento padrão (medidas do corpo) ou o comprimento da cabeça (medidas da cabeça).

\begin{tabular}{|c|c|c|c|c|c|}
\hline Medida & $\mathrm{N}$ & Mínimo & Máximo & Média & DP \\
\hline Comprimento padrão & 15 & 55,4 & 115,1 & & \\
\hline \multicolumn{6}{|c|}{ Porcentagem do comprimento padrão } \\
\hline Comprimento da cabeça & 15 & 23,7 & 28,2 & 25,9 & 1,422 \\
\hline Distância pré-anal & 15 & 69,7 & 73,4 & 71,5 & 1,233 \\
\hline Distância pré-dorsal & 15 & 60,9 & 65,6 & 63,1 & 1,059 \\
\hline Distância pré-pévica & 15 & 51,6 & 57,4 & 53,8 & 1,734 \\
\hline Distância pré-peitoral & 15 & 24,8 & 29,7 & 26,9 & 1,389 \\
\hline Base da nadadeira anal & 15 & 26,2 & 31,6 & 28,8 & 1,574 \\
\hline Base da nadadeira dorsal & 15 & 10,7 & 13,6 & 11,8 & 0,769 \\
\hline Comprimento do pedúnculo caudal & 15 & 9,2 & 12,2 & 10,9 & 0,840 \\
\hline Altura do pedúnculo caudal & 15 & 8,8 & 10,6 & 9,9 & 0,554 \\
\hline Altura do corpo na origem da nadadeira dorsal & 15 & 32,1 & 38,8 & 35,4 & 1,778 \\
\hline Maior altura do corpo & 15 & 35,5 & 42,3 & 39,5 & 1,682 \\
\hline Comprimento da nadadeira dorsal & 15 & 16,5 & 23,7 & 20,1 & 1,582 \\
\hline Comprimento da nadadeira pélvica & 15 & 14,7 & 18,1 & 16,4 & 0,898 \\
\hline Comprimento da nadadeira peitoral & 15 & 36,7 & 41,5 & 39,8 & 1,438 \\
\hline Comprimento da nadadeira anal & 15 & 13,1 & 19,7 & 16,4 & 1,756 \\
\hline $\begin{array}{l}\text { Distância entre a base do último raio da nadadeira } \\
\text { dorsal e a base da nadadeira adiposa }\end{array}$ & 15 & 14,9 & 18,2 & 16,6 & 1,000 \\
\hline \multicolumn{6}{|c|}{ Porcentagem do comprimento da cabeça } \\
\hline Comprimento do focinho & 15 & 19,6 & 25,2 & 22,6 & 1,611 \\
\hline Comprimento da maxila superior & 15 & 32,1 & 40,5 & 37,0 & 2,277 \\
\hline Diâmetro horizontal do olho & 15 & 26,2 & 36,8 & 31,6 & 2,813 \\
\hline Menor distância interorbital & 15 & 33,9 & 41,1 & 37,9 & 2,137 \\
\hline Comprimento da região pós-orbital da cabeça & 15 & 42,3 & 51,3 & 46,3 & 2,851 \\
\hline
\end{tabular}

A maior parte dos dados morfométricos obtidos para as duas espécies estão dentro dos apresentados por Malabarba (2004), com exceção de algumas medidas. A variação do comprimento do pedúnculo caudal de T. rotundatus apresentado pelo presente trabalho aumentou sua sobreposição com T. angulatus. As outras medidas cujas variações apresentadas pelo presente trabalho diferem do trabalho de Malabarba (2004) são o comprimento da maxila superior, o diâmetro horizontal do olho e a menor distância interorbital de ambas espécies. Esses novos valores também apresentam sobreposição entre $T$. angulatus e $T$. rotundatus. 
Os dados morfométricos não foram capazes de apresentar valores que permitissem diagnosticar T. angulatus e T. rotundatus como espécies distintas, no entanto, assim como no trabalho de Malabarba, 2004, os dados merísticos (tabela 3, 4 e 5) se mostraram capazes de permitir a identificação de exemplares como $T$. angulatus ou $T$. rotundatus.

Tabela 3: Dados merísticos de Triportheus angulatus e Triportheus rotundatus, juntamente com os dados para as mesmas espécies apresentados por Malabarba (2004).

\begin{tabular}{lcccc}
\hline & T.angulatus & T.rotundatus & $\begin{array}{l}\text { T. angulatus } \\
\text { (Malabarba, 2004) }\end{array}$ & $\begin{array}{l}\text { T. rotundatus } \\
\text { (Malabarba, 2004) }\end{array}$ \\
\hline $\begin{array}{l}\text { Escamas perfuradas na } \\
\text { linha lateral }\end{array}$ & $34-37$ & $30-33$ & $34-37$ & $28-34$ \\
\hline $\begin{array}{l}\text { Raios ramificados na } \\
\text { nadadeira anal }\end{array}$ & $25-30$ & $25-28$ & $26-31$ & $25-29$ \\
\hline $\begin{array}{l}\text { Rastros no ramo inferior } \\
\text { do primeiro arco branquial }\end{array}$ & $29-37$ & $25-31$ & $29-37$ & $24-32$ \\
\hline
\end{tabular}

Tabela 4: Distribuição do número de escamas perfuradas na linha lateral nos exemplares de Triportheus angulatus e T. rotundatus examinados pelo presente estudo.

\begin{tabular}{lcccccccc}
\hline $\begin{array}{l}\text { Escamas perfuradas } \\
\text { na linha lateral }\end{array}$ & 30 & 31 & 32 & 33 & 34 & 35 & 36 & 37 \\
\hline T. angulatus & - & - & - & - & 10 & 22 & 42 & 40 \\
T. rotundatus & 1 & 4 & 2 & 8 & - & - & - & - \\
\hline
\end{tabular}

Tabela 5: Distribuição do número de rastros no ramo inferior do primeiro arco branquial nos exemplares de Triportheus angulatus e T. rotundatus examinados pelo presente estudo.

\begin{tabular}{cccccccccccccc}
\hline Rastros branquiais & 25 & 26 & 27 & 28 & 29 & 30 & 31 & 32 & 33 & 34 & 35 & 36 & 37 \\
\hline T. angulatus & - & - & - & - & 1 & 2 & 8 & 18 & 21 & 26 & 25 & 9 & 4 \\
T. rotundatus & 1 & 1 & 3 & 7 & 1 & - & 2 & - & - & - & - & - & - \\
\hline
\end{tabular}

O exame de mais material proveniente da bacia Amazônica mostrou que os dados merísticos são consistentes para diagnosticar T. angulatus e T. rotundatus como espécies distintas, como proposto por Malabarba (2004). O número de raios ramificados da nadadeira anal não ajuda o diagnóstico das duas espécies pois apresenta grande sobreposição. Já com relação ao número de rastros no ramo inferior 
do primeiro arco branquial, a maioria dos exemplares apresentaram valores fora da zona de sobreposição (tabela 5). O número de escamas perfuradas na linha lateral não apresentou sobreposição nenhuma nos exemplares examinados, sendo talvez a melhor medida para diagnosticar as duas espécies, apesar dos dados do trabalho de Malabarba (2004:170) (tabela 3) mostrarem sobreposição nesse atributo.

Material examinado (132 lotes; 1396 exemplares; 30,1-161,5 mm CP)

BRASIL: Acre: MZUSP 20511, 1, 104,9 mm CP; Seringal União, baixo rio Acre. MZUSP 30716, 4; Rio Tarauacá. MZUSP 35792, 1; Igarapé da Maternidade. MZUSP 49789, 1; Lago Amapá. MZUSP 49797, 1, 141,4 mm CP; Lago Corredeira. MZUSP 49823, 2, 92,7-163,8 mm CP; Rio Acre, Colocação. MZUSP 50302, 1; Rio São João, Ponto3, Depósito. MZUSP 50304, 1; Rio São João, Ponto 4, Foz. Amazonas: MZUSP 5870, 2; Boca do lago Preto, Rio Solimões. MZUSP 5908, 15; Lago Jacaré, margem direita do rio Solimões, acima de Manacapuru. MZUSP 5936, 7; Boca do Rio Purus. MZUSP 6062, 10, 148,6-160,4 mm CP; Lago Rei, ilha do Careiro. MZUSP 6092, 3; Lago Puraquequara, boca do rio Puraquequara. MZUSP 6376, 3; Lago Baruri, Rio Purus. MZUSP 6532, 3; Lago Manacapuru. MZUSP 6948, 78, 69,1-149,4 mm CP; Rio Madeira, 25 km abaixo de Nova Olinda. MZUSP 7522, 19; Paraná do Urucará. MZUSP 7582, 3, 104,6-120,8 mm CP; Paraná do Mocambo, perto de Parintins. MZUSP 13425, 1; Rio Amazonas. MZUSP 13426, 1; Rio Amazonas. MZUSP 13427, 1; Rio Amazonas. MZUSP 13428, 1; Rio Amazonas. MZUSP 19434, 1, 110,0 mm CP; Benjamin Constant. MZUSP 19769, 1; Lago Janauacá, margem direita do rio Solimões. MZUSP 20004, 8, 48,2-56,8 mm CP; Tefé. MZUSP 20019, 6, 121,8-127,9 mm CP; Rio Solimões. MZUSP 20033, 1; Rio Içapo, foz do rio Jutaí. MZUSP 20043 1; Ilha Xibeco, rio Solimões, acima da boca do rio 
Jutaí. MZUSP 20059, 3; Boca do Rio Iça, Santo Antônio do Iça. MZUSP 20073, 1, 108,6 mm CP; Rio Solimões, próximo da ilha Baruruá, acima da boca do rio Jutaí. MZUSP 20084, 3; Igarapé Tomé, Ati-Paraná, NW da Fonte Boa. MZUSP 20090, 3, 53,5-54,6 mm CP; Lago Buiuçu, Ati-Paraná, NW de Fonte Boa. MZUSP 20094, 1; Igarapé Tucuxi, Ati-Paraná, NW de Fonte Boa. MZUSP 20101, 10; Igarapé Manduaçu, paraná de Iupiá, NW de Fonte Boa. MZUSP 20124, 8, 49,6-65,5 mm CP; Igarapé em Jacaré, próximo de Fonte Boa. MZUSP 20132, 1, 137,9 mm CP; Rio Solimões. MZUSP 20149, 13, 41,1-66,9 mm CP; Poça na ilha Sorubim, rio Solimões, acima de Coari. MZUSP 20156, 58, 53,4-100,7 mm CP; Braço do rio Solimões, ilha Sorubim, acima de Coari. MZUSP 20163, 22, 56,6-137,1 mm CP; Rio Solimões. MZUSP 20386, 6; Lago Janauacá e arredores, rio Solimões. MZUSP 20455, 4; Rio Purus. MZUSP 20516, 2, 110,2-118,3 mm CP; Pauini. MZUSP 20519, 1; Boca do Ituxi. MZUSP 20523, 2; Boca do Paciá. MZUSP 27312, 1, 103,5 mm CP; Foz do rio Jutaí, rio Solimões. MZUSP 27314, 7, 82,1-101,5 mm CP; Costa Japão, baixo rio Japurá. MZUSP 27315, 8; Foz do rio Iça, Rio Solimões. MZUSP 27316, 14, 120,8141,8 mm CP; Costa do Capacete, Rio Solimões. MZUSP 35551, 21; Igarapé Joari, Humaitá. MZUSP 49518, 3; Rio Acre. MZUSP 49552, 1, 92,3 mm CP; Rio Acre, junto ao lago Lua Nova. MZUSP 58983, 5; Rio Negro, Anavilhanas, Pauzada. MZUSP 59237, 10, 65,3-69,5 mm CP; L. Murumuru, plantação de juta, Janauacá. MZUSP 59243, 5, 57,7-83,4 mm CP; Lago Janauari, perto de sua desembocadura. MZUSP 67926, 3 (apenas 1 no frasco), 129,6 mm CP; Lago Pirapora, margem esquerda do rio Acre, AM 16 km município de Porto Acre. MZUSP 68985, 3; Lago Cuiuanã, rio Purus. MZUSP 74479, 3, 71,8-90,8 mm CP; Lagoa junto ao canal do lago Janauacá, margem direita do rio Solimões. MZUSP 74585, 2; Lagoa na Ilha Jacurutu, em frente à boca do Janauacá. MZUSP 75405, 1, 81,4 mm CP; Igarapé na 
margem direita do canal do Lago Janauacá, marg. direita do Rio Solimões. MZUSP 75718, 1, 106,9 mm CP; Lago Janauari, Primeira Olaria. MZUSP 75719, 5; Ressaca da Ilha de Marchantaria. MZUSP 75720, 2; Camaleão, Ilha da Marchantaria. MZUSP 75721, 10; Lago Terra Preta, Janauari. MZUSP 75722, 4; Lago Terra Preta, Janauari. MZUSP 75723, 39, 35,1-100,5 mm CP; MZUSP 75724, 2; Ressaca da Ilha de Marchantaria. MZUSP 75725, 6; Lago Janauari, Pequena Casa quase frente da Olaria. MZUSP 75726, 8, 77,7-129,0 mm CP; Ressaca da Ilha de Marchantaria. MZUSP 75727, 27, 35,3-81,8 mm CP; Furo entre lago Murumuru e Paraná de Janauacá. MZUSP 75728, 2, 89,7-99,4 mm CP; Lago Janauari, perto da sua desembocadura. MZUSP 75730, 380, 20,8-66,2 mm CP; Lago Murumuru, perto do cural do INPA, Janauacá. MZUSP 75731, 13, 81,2-103,3 mm CP; Lago Janauari, Pequena Casa quase frente da Olaria. MZUSP 75732, 46; Lago Terra Preta, Janauari. MZUSP 75733, 1, 116,6 mm CP; Paraná do Janauacá, entrada do Lago do Castanho. MZUSP 75734, 71, 54,1-82,2 mm CP; Furo entre Lago Murumuru e Paraná do Janauacá. MZUSP 75736, 95, 46,9-65,5 mm CP; Ressaca da Ilha de Marchantaria. MZUSP 75737, 6; Lago Terra Preta, Janauari. MZUSP 75738, 32; Ressaca da Ilha de Marchantaria. MZUSP 75739, 50, 37,3-50,3 mm CP; Lago Murumuru, plantação de juta, Janauacá. MZUSP 100175, 2, 81,4-85,7 mm CP; Lagoa mg. esq. do canal do lago Janauacá, m. dir. rio Solimões. INPA 19301, 2, 89,7-94,0 mm CP; Lago Amanã, Boca do Rio Tefé. INPA 19304, 2, 70,4-104,8 mm CP; Rio Solimões, ilha do Içe, cerca Tefé. INPA 5826, 9, 89,3-117,3 mm CP; Rio Solimões, lago do Castanho. INPA 10690, 1, 161,3 mm CP; Rio Amazonas, paraná do Rei, ilha do Careiro. INPA 29058, 1, 117,6 mm CP; RDS Uacari, calha do rio Juruá, comunidade mandioca. INPA 29065, 1, 141,8 mm CP; RDS Uacari, calha do rio Juruá. Boca do Igarapé Anaxiqui, comunidade Boa Vista. INPA 29080, 1, 115,0 mm CP; RDS Uacari, calha do rio Juruá, boa do Igarapé Xué, 
comunidade Santo Antônio do Brito. INPA 29750, 2, 116,5-137,4 mm CP; RDS Uacari, Lago próximo a comunidade Xibauá. INPA 29766, 1, 113,8 mm CP; RDS Uacari, Igarapé Bauana. INPA 25070, 4, 137,9-161,5 mm CP; Rio Madeira, ig. do Lago Xadá, ao Paraná da Comunidade Realeza. Pará: MZUSP 5445, 5, 124,1-136,6 mm CP; Rio Trombetas. MZUSP 5706, 1; Rio Tapajós. MZUSP 8253, 1; Rio Trombetas. MZUSP 8522, 1; Rio Tapajós. MZUSP 9175, 3; Rio Maicá. MZUSP 9229, 1; Rio Tapajós. MZUSP 9468, 1, 140,9 mm CP; Taperinha, abaixo de Santarém. MZUSP 9502, 13, 49,2-82,1 mm CP; Rio Amazonas, Monte Alegre. MZUSP 45802, 25, 49,6-57,8 mm CP; Monte Alegre. MZUSP 57691, 3, 94,9-110,5 mm CP; Rio Amazonas. MZUSP 59156, 11; Rio Trombetas, 20km acima da boca, beira do rio. MZUSP 59157, 2; Rio Trombetas, Cuminá, Beira do rio. MZUSP 92773, 1, 109,8 mm CP; Lago do Maiacá, na margem direita do Rio Amazonas, próximo à Santarém. INPA 3404, 1, 143,0 mm CP; Rio Cuminá, lago Salgado. Rondônia: MZUSP 14013, 7; Rio Machado, (boca). MZUSP 30510, 3; Rio Madeira, Calama, paraná do Caraparu. MZUSP 30512, 1, 114,1 mm CP; Rio Machado, lago do Cururu. MZUSP 30515, 6, 68,2-78,4 mm CP; Rio Madeira, encontro com o rio Machado. MZUSP 30517, 8, 76,6-128,6 mm CP; Rio Madeira, várzea de Calama, lago de Repartimento. MZUSP 30518, 2; Rio Madeira, boca do rio Machado. MZUSP 30717, 5; Rio Madeira, Cachoeira do Teotônio. MZUSP 58961, 1, 137,4 mm CP; Rio Madeira, at Calama. INPA 10599, 2, 159,5-161,4 mm CP; Rio Jamari, lago Espanha, cerca de $15 \mathrm{~km}$ a jusante UHE Samuel. INPA 10692, 5, 84,8-112,3 mm CP; Rio Guaporé, Surpresa, margem. INPA 16182, 6, 81,1-96,6 mm CP; Rio Mamoré, em frente Guajará Mirim. Roraima: MZUSP 19386, 1; Rio Mucajaí, ao sul de Boa Vista. MZUSP 58864, 1; Igarapé do Cujobim, rio Branco, em frente da ilha de Maracá. 
MZUSP 59016, 1; Rio Branco, Cachoeira do Bem-Querer, em igarapé 2 km acima da cachoeira.

PERU: Loreto: MZUSP 26439, 30,1 mm CP; Supaycocha, Jenaro Herrera. MZUSP 4506, 1, 121,2 mm CP; Rio Pacaya. MZUSP 15268, 1, 90,8 mm CP; Rio Samiria. MZUSP 15270, 2; Rio Pacaya, Bretaña. MZUSP 26454, 2, 111,0-118,2 mm CP; Rio Ucayali, Bahuanisho. Napo: Rio Yasuni, confluência cone 1 rio Jatuncocha. Ucayali: MZUSP 26251, 3, 59,5-64,9 mm CP; Yarinacocha, Pucallpa, Prov. Coronel Portillo. MZUSP 25999, 3, 60,5-78,1 mm CP; Lobococha, Masisea, Prov. Coronel Portillo. MZUSP 26000, 5, 32,2-37,1 mm CP; Lobococha, Masisea, Prov. Coronel Portillo. MZUSP 26164, 3, 59,9-105,0 mm CP; Rio Ucayali, Bagazan. MZUSP 26716, 14; Rio Ucayali, Bagazan. MZUSP 26716, 1, 127,7 mm CP; Rio Ucayali, Pucallpa. MZUSP 26290, 7; Cashibococha, Pucallpa, Prov. Coronel Portillo. SEM LOCALIDADE: INPA 13888, 3, 70,5-81,9 mm CP.

Triportheus auritus (Valenciennes in Cuvier \& Valenciennes, 1850)

(Figura 5) 


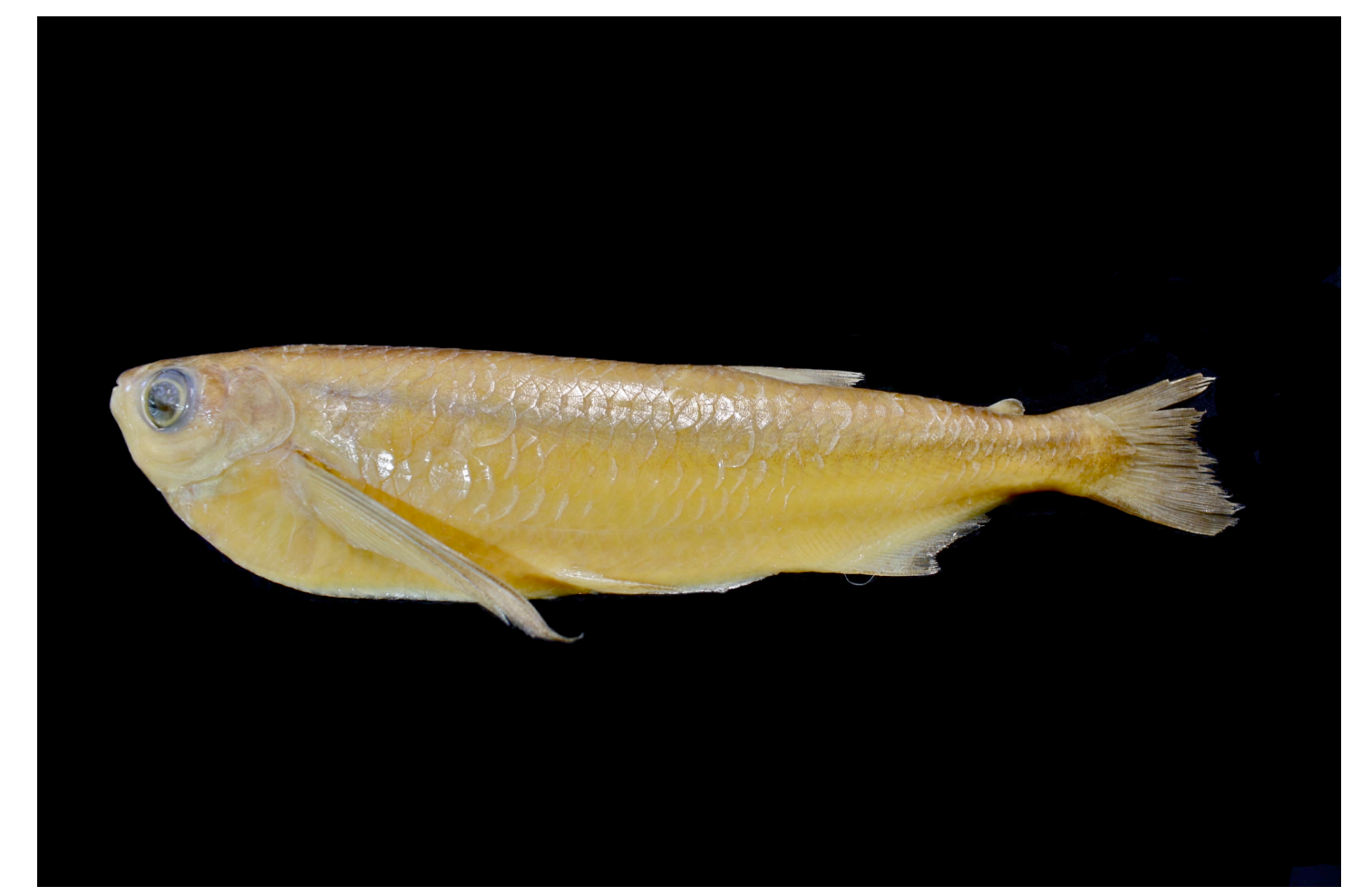

Figura 5: Triportheus auritus, MZUSP 6669, 150,0 mm CP, Brasil, Amazonas, Rio Negro, arredores de Manaus.

Essa espécie está amplamente distribuída nos rios da bacia amazônica incluindo toda extensão dos rios Amazonas e Solimões, além dos rios Araguari, Xingu, Trombetas, Tapajós, Negro, Branco e Ambiyacu, e em rios das bacias Tocantins-Araguaia e Orinoco (figura 6). Na figura apresentada por Malabarba (2004: fig. 8) a localidade do neótipo está representada próxima do rio Matupiri, afluente do rio Madeira. Contudo, o ponto correto deveria estar próximo à Anavilhanas, no rio Negro, conforme a informação da localidade do exemplar designado pela autora como neótipo (MZUSP 58859, Brasil, Amazonas, Rio Negro, Anavilhanas. Malabarba 2004:179). 


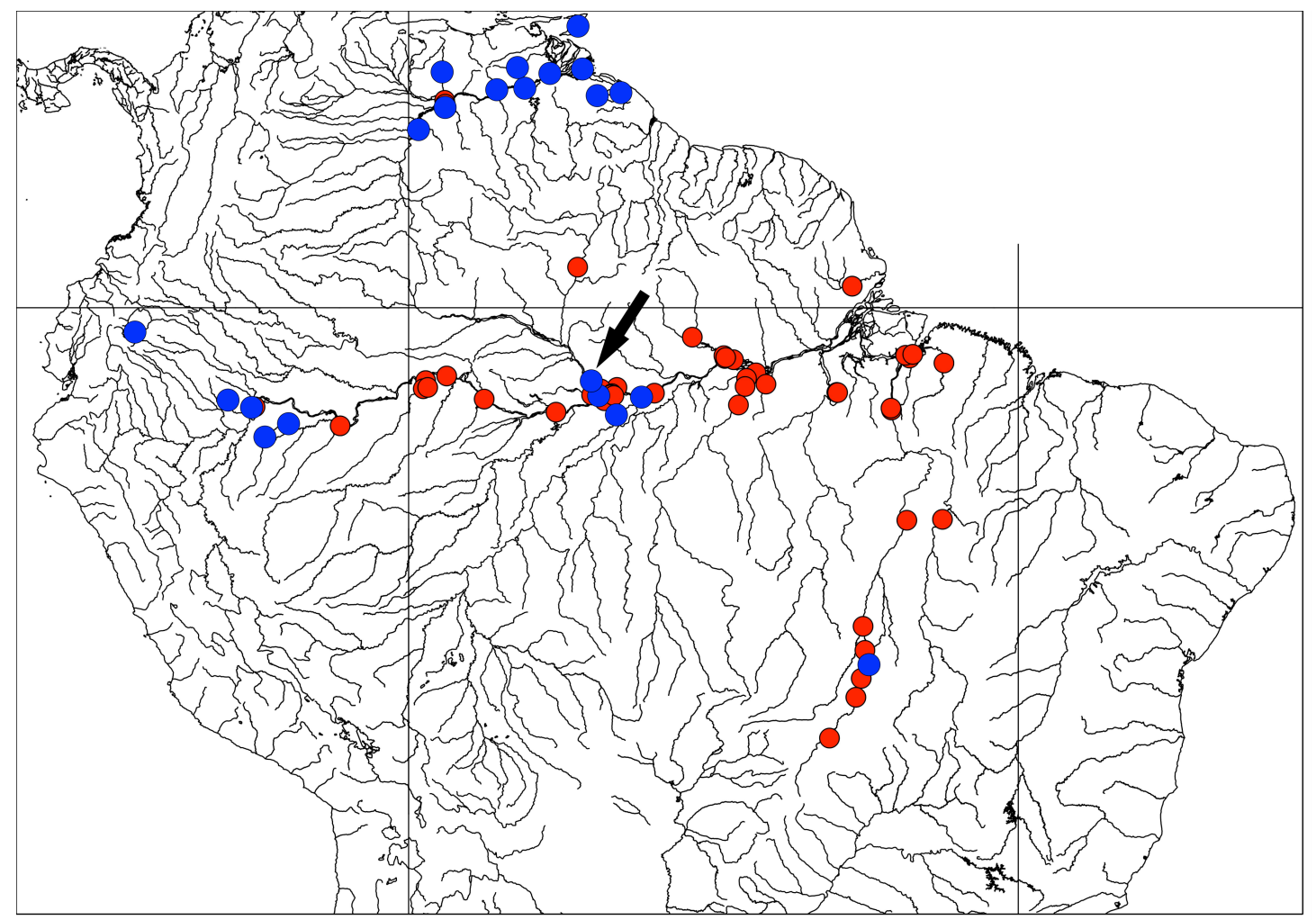

Figura 6: Distribuição geográfica de Triportheus auritus. Círculos azuis indicam localidades fornecidas por Malabarba (2004: fig. 8). Círculos vermelhos representam lotes examinados no presente estudo. Seta indica localidade tipo.

\section{Comentários sobre Triportheus auritus}

Triportheus auritus foi registrada por Malabarba (2004: fig. 8) para os rios Araguaia, Amazonas, Negro, Solimões, Orinoco, Yasuni, Nanay e Ambiyacu, além de Trinidade Tobago. O presente estudo registrou diversos pontos de ocorrência ao longo dos rios Solimões, Amazonas e baixo Tocantins. Além disso, de T. auritus também ocorre nos rios Araguari, Capim, Xingu, Tapajós, Trombetas e Branco. A adição dessas localidades a informação da distribuição geográfica da espécie mostrou que existe continuidade entre as áreas de ocorrência definidas por Malabarba (2004:fig. 8).

Material Examinado (72 lotes, 417 exemplares; 32,8-230,6 mm CP) 
BRASIL: Amapá: MZUSP 59005, 5; Rio Araguari. MZUSP 59010, 6, 166,8207,9 mm CP; Rio Araguari. Amazonas: MZUSP 87132, 14, 139,8-208,2 mm CP; Rio Negro, Anavilhanas. MZUSP 6091, 2, 178,6-205,8 mm CP; Lago Puraquequara, boca do rio Puraquequara. MZUSP 6179, 4, 212,6-225,4 mm CP; Igarapé Jaraqui, margem esquerda do rio Negro, acima de Manaus. MZUSP 6669, 2, 124,4-148,3 mm CP; Rio Negro, arredores de Manaus. MZUSP 13421, 1; Rio Amazonas. MZUSP 13422, 1; Rio Amazonas. MZUSP 13423, 1; Rio Amazonas. MZUSP 13424, 1; Rio Amazonas. MZUSP 19766, 61, 19,0-41,9 mm CP; Lago Janauacá, margem direita do rio Solimões. MZUSP 19785, 1, 36,1 mm CP; Lago Janauacá e vizinhanças. MZUSP 20021, 3; Rio Solimões. MZUSP 27317, 2; Costa do Capacete, Rio Solimões. MZUSP 27319, 1, 192,2 mm CP; Rio Solimões, Foz do Rio Japurá. MZUSP 30516, 1; Rio Tefé, lago. MZUSP 56577, 1, 197,1 mm CP; Rio Solimões, 27,2 km abaixo do Paraná Porto Alegre. MZUSP 59095, 11, 138,3-187,4 mm CP; Rio Negro, Anavilhanas, Pauzada. MZUSP 59150, 3, 150,7-173,4 mm CP; Lago Pantaleão. MZUSP 59151, 3, 184,4-204,3 mm CP; Parana do Castelo. MZUSP 59153, 1, 205,16 mm CP; Igarapé Urumutian. MZUSP 75711, 6, 97,3-110,6 mm CP; Lago Janauari, primeira Olaria. MZUSP 75712, 7, 110,4-146,7 mm CP; Camaleão, Ilha de Marchantaria. MZUSP 75714, 21, 36,6-41,0 mm CP; Ressaca da Ilha da Marchantaria. MZUSP 75715, 58, 32,8-43,3 mm CP; Ressaca da Ilha de Marchantaria. MZUSP 75716, 2, 89,7-92,9 mm CP; Lago Janauari, pequena casa quase frente da Olaria. MZUSP 75717, 20, 50,9-75,7 mm CP; Ressaca da Ilha de Marchantaria. MZUSP 6531, 8, 88,5-174,5 mm CP; Lago Manacapuru. MZUSP 7038, 11; Rio Canumã. MZUSP 9651, 2 (só tinha 1 no frasco), 70,0 mm CP; Lago Supiá, em frente a Codajás. MZUSP 20032, 1, 161,6 mm CP; Rio Içapó, foz do rio Jutaí. MZUSP 59014, 10, 144,3-194,9 mm CP; Rio Negro, Anavilhanas. MZUSP 
59238, 3, 88,2-92,5 mm CP; Lago Murumuru, perto do cural do INPA, Janauacá. MZUSP 59241, 12, 47,0-50,3 mm CP; Ressaca da Ilha de Marchantaria. INPA 13923, 4, 58,8-157,7 mm CP; Rio Solimões, Ilha da Marchantaria. Mato Grosso: MZUSP 54449, 1, 127,5 mm CP; Rio Cristalino. MZUSP 62995, 2, 209,50-210,10 mm CP; Rio Araguaia, Ouro Fino, 30 km a jusante de Barra do Garças. MZUSP 63022, 2, 197,6-203,9 mm CP; Rio Araguaia, Pontal (perto da foz do rio das Mortes). MZUSP 52312, 1, 226,10 mm CP; Rio Araguaia. MZUSP 52392, 7, 198,1-229,3 mm CP; Rio Araguaia, Bandeirantes. MZUSP 53593, 2, 192,9-195,1 mm CP; Rio Araguaia, Cocalinho. MZUSP 62516, 1, 198,8 mm CP; Rio Araguaia, próximo a ilha do Biratã. Pará: MZUSP 3532, 1; Rio Tapajós. MZUSP 5626, 3; Lago Paru. MZUSP 8254, 1; Rio Trombetas. MZUSP 9467, 2; Taperinha, abaixo de Santarém. MZUSP 20280, 1; Rio Tapajós, Barreirinha. MZUSP 20321, 2; Ilha Tapaiuna, Rio Tapajós. MZUSP 20334, 2; Lago Trocará, abaixo de Tucuruí, rio Tocantins. MZUSP 20366, 2; Lagoas na margem do rio Tocantins, perto de Tucuruí. MZUSP 20598, 7; Vila Santana, Rio Capim. MZUSP 20602, 1; Vila Maiauatá, Baia de Maiauatá. MZUSP 20608, 1; Braço do igarapé São Lourencinho, Furo de Panaquera. MZUSP 20612, 16; Igarapé Sororoca, Furo de Panaquera. MZUSP 20614, 2; Igarapé Coelho, Boca do Tocantins. MZUSP 20639, 9; Lago às margens do igarapé Espírito Santo, entre Baião e Tucuruí, rio Tocantins. MZUSP 59009, 5, 192,2-230,6 mm CP; Rio Xingu, Belo Monte. MZUSP 59011, 5, 181,0-201,4 mm CP; Rio Xingu, Belo Monte. MZUSP 6250, 1, 79,9 mm CP; Obidos. MZUSP 9512, 1; Rio Tapajós. MZUSP 20327, 2; Igarapé Urubu, perto do Posto de Trocará, rio Tocantins. MZUSP 20607, 1, 92,3 mm CP; Igarapé Jacarequara. MZUSP 20647, 1, 100,0 mm CP; Igarapé Muru, rio Tocantins, abaixo de Tucuruí. MZUSP 20656, 2; Lagoa na margem do igarapé Muru, rio Tocantins, abaixo de Tucuruí. MZUSP 59155, 7, 113,5-204,5 mm CP; Rio 
Trombetas, $20 \mathrm{~km}$ acima da boca, beira do rio. MZUSP 106050, 1, 222,6 mm CP; Rio Xingu. INPA 5824, 2, 178,5-195,3 mm CP; Rio Tocantins, Capuerana. INPA 16237, 1, 217,3 mm CP; Rio Trombetas, cachoeira Porteira, a jusante da cachoeira. Roraima: MZUSP s/n (desdobrado de MZUSP 74117), 6; Rio Branco, Marará, Praia. Tocantins: MZUSP 86157, 1, 186,1 mm CP; Rio Manoel Alves Pequeno, c. $10 \mathrm{~km}$ acima da foz no rio Tocantins.

PERU: Loreto: MZUSP 15249, 1, 51,6 mm CP; Padre Isla (Rio Amazonas).

VENEZUELA: Bolivar: MZUSP 98044, 8; Rio Orinoco, Puerto Cedeño. MZUSP 98047, 10; Laguna de Castilleros (marginal ao Rio Orinoco), Estação de Aquicultura da Universidad de Oriente.

Triportheus brachipomus (Valenciennes in Cuvier \& Valenciennes, 1850) Figura 7

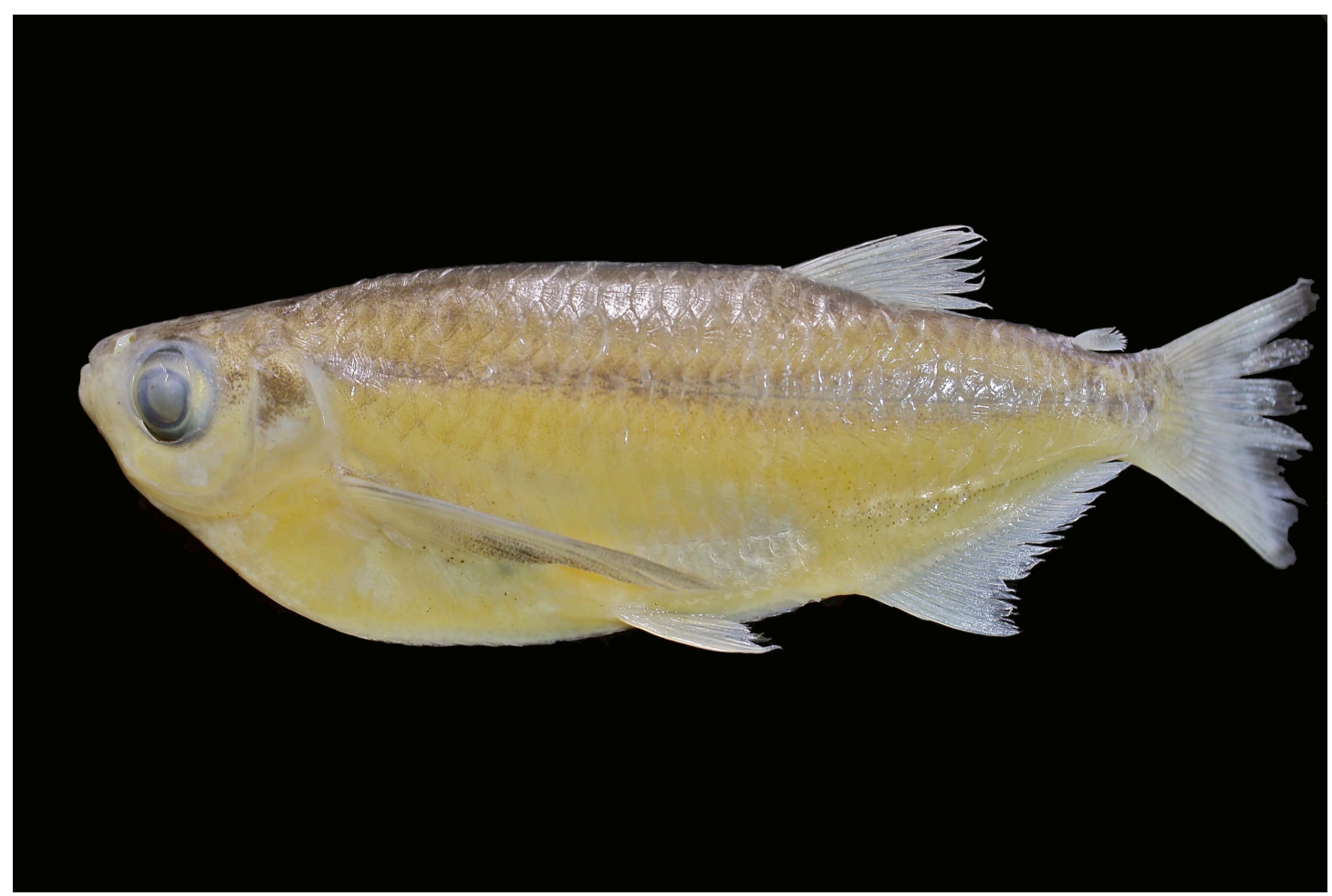

Figura 7: Triportheus brachipomus, MZUSP 104764, 99,0 mm CP, Brasil, Amapá, Laranjal do Jari, Igarapé Três bocas, afluente do Pacanari, a jusante da cachoeira Santo Antônio. 
Essa espécie está distribuída pelos rios Essequibo, Demerara, Courantyne, Maroni, Araguari, Xingu, Trombetas, Tapajós, Jari e Negro (figura 8). Localidade tipo: próximo da cidade de Mana, na Guiana Francesa.



Figura 8: Distribuição geográfica de Triportheus brachipomus. Círculos azuis indicam localidades fornecidas por Malabarba (2004: fig. 2). Círculos vermelhos representam lotes examinados no presente estudo. Seta indica localidade tipo.

Comentários sobre Triportheus brachipomus

Essa espécie foi registrada por Malabarba (2004: fig. 2) para os rios Orinoco, Essequibo, Demerara, Courantyne, Maroni e Araguari (porém apesar dos lotes do Rio Orinoco terem sido listados pela autora como material examinado, as localidades de origem dos exemplares não foram incluídas no mapa apresentado por ela). Além 
disso, T. brachipomus também ocorre nos rios Xingu, Trombetas, Tapajós, Jari e Negro. Na chave de identificação apresentada pela autora a espécie foi incluída apenas na seção para as espécies de Triportheus do rio Orinoco e dos rios costeiros entre os rios Essequibo e Araguari, uma vez que a espécie estaria restrita a esses rios. Assim, com o registro de ocorrência da espécie nos rios da bacia Amazônica a chave de identificação de Malabarba (2004:170-171) teria que ser revista.

Material examinado (30 lotes, 797 exemplares; 20,0-214,4 mm CP)

BRASIL: Amapá: MZUSP 58855*, 13, 142,6-175,9 mm CP; Rio Araguari, canal de rio. MZUSP 104877, 1, 117,9 mm CP; Laranjal do Jari. Rio Iratapuru, afl. da margem esquerda do Jari, na comunidade Iratapuru. MZUSP 104764, 1, 99,0 mm CP; Laranjal do Jari. Igarapé Três Bocas, afl. do Pacanari, a jusante da cachoeira Santo Antônio. Amazonas: MZUSP 43451, 1, 72,1; Rio Negro, Tapera. MZUSP 59006, 6, 197,5-201,9 mm CP; Rio Negro, São Gabriel da Cachoeira, igapó. MZUSP 59017, 8; Rio Negro, Anavilhanas, igapó. MZUSP 59096, 1, 171,7 mm CP; Rio Negro, logo abaixo do rio Daraá, Pedral. MZUSP 59097, 1, 179,5 mm CP; Rio Negro, ilha Marimari, igapó. MZUSP 59098, 1, 207,6 mm CP; Alto rio Negro, encontro do igarapé do Ibará. MZUSP 59101, 10, 182,0-195,0 mm CP; Alto Rio Negro, encontro do igarapé do Ibará. MZUSP 59102, 1, 207,5 mm CP; Rio Negro, Marauiá, cachoeira do BichoAçu. MZUSP 59103, 3, 212,9-214,4 mm CP; Igapó de água preta na confluência dos rios Marauiá e Negro. MZUSP 62222, 1, 71,7 mm CP; Lagoa em ilha do Rio Negro, Paricatuba. INPA 3311, 5, 174,4-178,2 mm CP; Rio Pitinga, Pé da barragem de Paranapanema. Mato Grosso: MZUSP 84900, 1; Rio Juruena, cerca de $1 \mathrm{~km}$ acima da desembocadura do rio Arinos, alagamento do rio na margem da estrada. Pará: MZUSP 20279, 1, 169,4 mm CP; Rio Tapajós, Barreirinha. MZUSP 92723, 40, 25,198,4 mm CP; Rio Tapajós, margem direita, em Pimental. MZUSP 23708, 634, 20,0- 
48,9 mm CP; Poça do rio Tapajós, Barreirinha, próximo a São Luís. MZUSP 20275, 7; Rio Tapajós, lago na ilha em frente a Monte Cristo. MZUSP 25360, 1; Margem direita do rio Tapajós, defronte a Pimental, (PARNA). MZUSP 25514, 1, 197,5 mm CP; Igarapé Uruá (ou da Boa Vista) afluente da margem esquerda do rio Tapajós, junto à ponte do km 62 da BR 230, PARNA. MZUSP 30743, 1; Rio Xingu, Belo Monte. MZUSP 59152, 1; Rio Tapajós, São Luis acima de Itaituba, Pedral. MZUSP 34069, 3, 72,6-76,9 mm CP; Xingu, Belo Monte. INPA 5424, 4, 150,8-180,7 mm CP; Rio Trombetas, Rio Cachorro. INPA 5845, 5, 136,8-171,6 mm CP; Rio Trombetas, cachoeira Porteira. INPA 3401, 1, 145,07 mm CP; Rio Trombetas, jusante da cachoeira Vira-Mundo. MNRJ 35223, 3, 35,0-60,8 mm CP; Praia do Pge, rio Xingu a montante de Altamira e igarapé Panela. MNRJ 35224, 4, 37,4-54,9 mm CP; Rio Xingu, margem esquerda no porto da balsa de São Felix para Vila Central. MNRJ 35225, 35, 85,9-109,4 mm CP; Rio Xingu, na confluência com o rio Fresco.

\section{Triportheus culter (Cope, 1872)}

(Figura 9)

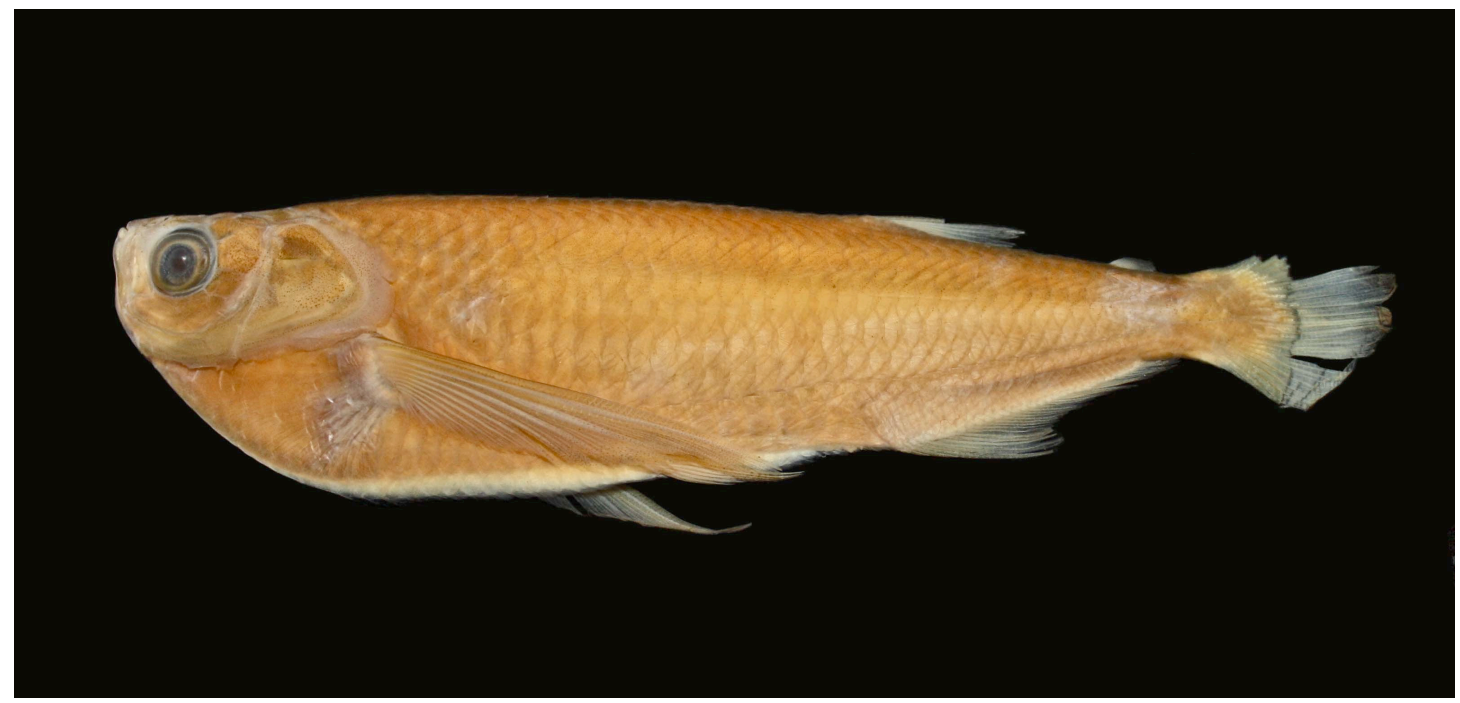

Figura 9: Triportheus culter, MZUSP 26717, 139,1 mm CP, Peru, Ucayali, Rio Ucayali, Pucallpa. 